\title{
Z Research Suare \\ MicroRNA-29c-3p in Dual-Labeled Exosome is Potential Marker of Alzheimer's Disease
}

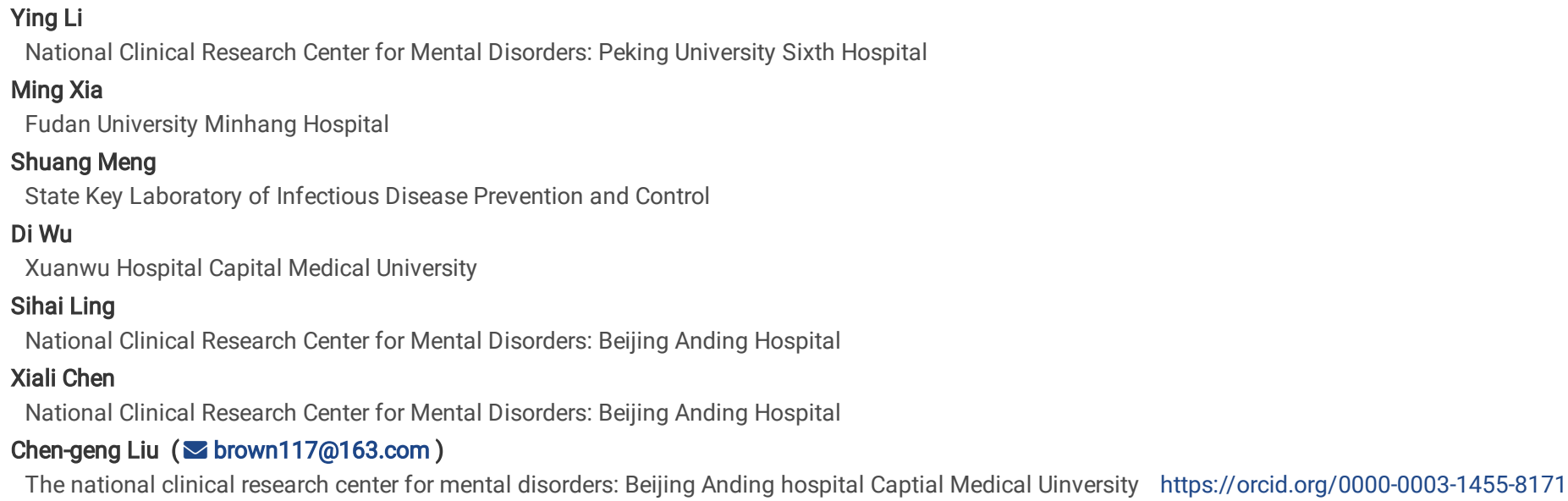




\section{Abstract}

Objective

We aimed to establish a method to determine whether peripheral blood NCAM/amphiphysin 1 dual-labeled exosomal proteins and microRNA might be serve as a marker for the diagnosis of Alzheimer's disease.

Methods

It was a multicenter study using a two-stage design. The subjects included $45 \mathrm{SCD}, 50 \mathrm{aMCl}, 40 \mathrm{AD}$ patients, and 30 controls in the discovery stage; the results were confirmed in the verification stage ( $47 \mathrm{SCD}, 45 \mathrm{aMCl}, 45 \mathrm{AD}$, and 30 controls). Peripheral blood NCAM single-labeled and NCAM/amphiphysin 1 doublelabeled exosomes were captured and detected by immunoassay, respectively.

Results

Levels of $A \beta 42, A \beta_{42 / 40}$, Tau, P-T181-tau, and miR-29c-3p in plasma NCAM single-labeled and NCAM/amphiphysin 1 double-labeled exosomes of the aMCI and $A D$ groups were significantly higher than those of the SCD group, control group and VaD group (all $P<0.05$ ). The levels of $A \beta 42$ and miR-29c-3p in peripheral blood NCAM/amphiphysin 1 dual-labeled exosome was higher than that in the control and VaD groups (all $P<0.05)$. The levels of exosomal Aß42, $\mathrm{A} \beta_{42 / 40}$, Tau, P-T181-tau, and miR-29c-3p in peripheral blood were correlated with that in CSF (all $P<0.05$ ).

Conclusion

This study first provided a method for sorting specific surface marker exosomes using a two-step immune capture technology. The plasma NCAM/amphiphysin 1 dual-labeled exosomal $A \beta_{42 / 40}$ and miR-29c-3p had potential advantages in the diagnosis of SCD.

\section{Introduction}

Diagnosis of Alzheimer's disease (AD) requires physical signs, psychological tests, syndrome judgment, and biomarker testing. AD biomarkers can generally be divided into two categories: "imaging markers" and "clinical laboratory diagnostic markers". In vivo diagnostic imaging markers such as amyloid PET (positron emission computed tomography) imaging have good diagnostic specificity on AD, and it also has potential value in the diagnosis of SCD, however the expensive costs of PET limit its application as a routine inspection item [1,2]. At present, there are no drugs that can effectively treat patients with DAT, but patients in the middle and early stages such as amnestic mild cognitive impairment (aMCl) and subjective cognitive decline (SCD) could avoid or delay the development of the disease through a series of treatments $[3,4]$. Therefore, it is more important to make a clear diagnosis in the early stages when $A \beta$ has not yet significantly accumulated [5]. Recent studies have shown that the increase and aggregation of amyloid $\beta$-protein (A $\beta$ ) outside the cell may not be the earliest molecular changes in $A D$ : a series of molecular biological changes have begun to occur in neuronal cells before the occurrence of $A \beta$ aggregation that can be detected by PET, which are considered to be the earliest (ultra-early) changes that lead to the occurrence, progression and clinical onset of $A D$ [5-7]. In recent years, with the conclusion of a number of large-scale long-term longitudinal studies, the clinical application value of multiple new familial $A D$ ultra-early diagnostic markers has been initially clarified. For example, molecules such as peripheral blood neurofilament light chain (NfL) are expected to provide early warning of the onset of familial AD with presenilin 1 mutant 10-15 years in advance, so as to achieve ultra-early early warning [8]. However, for sporadic AD, which accounts for $90 \%$ of the total number of $A D$, there is still a lack of effective peripheral blood ultra-early warning biomarkers.

CSF is in direct contact with the brain, and the material exchange with the brain is more direct than that of peripheral blood and other body fluids. Therefore, CSF is considered to be a "clinical laboratory diagnostic biomarker" specimen that can diagnose AD and other neurological diseases more specifically and earlier [2]. However, because CSF needs to be collected invasively by lumbar puncture, there is a certain clinical risk, and patient acceptance is low, and it is difficult to carry out routine and large-scale development. Due to the existence of the blood-brain barrier (BBB) transport mechanism, CSF and peripheral blood can also exchange certain substances. In recent years, a series of studies have suggested the possibility of using peripheral blood as a specimen source for $A D$ diagnostic markers. In addition, peripheral blood collection is less traumatic and patient acceptance is high. If markers with high disease diagnosis efficiency can be found, it will greatly promote the AD marker routine detection and effectively increase the AD detection rate. However, facts have proved that some potential AD markers (such as proteins, miRs, etc.) that have greater clinical value in CSF, when directly detected in peripheral blood, have unsatisfactory diagnostic performance such as positive rates. The reason may be the BBB transport process leads to changes in its "quality" or "quantity" or interference caused by the secretion of the same or similar substances in other tissues and organs of the body [2, 9].

At present, more researched are centered on the non-invasive approaches of the AD early diagnosis or warning. The series of protein biomarker panels, metabolomics biomarker panels, and nucleic acid biomarker panels were initially clarified [10]. MicroRNAs (miRs) are a class of small (18-25 nucleotides), single-stranded non-coding RNAs involved in the post-transcriptional regulation of gene expression. In recent years, miRs have been shown to play important roles in several diseases, such as cancer, cardiovascular disease, and diabetes, as well as central nervous system diseases. Because of their stable character, altered miRs in tissues and organs may lead to the deregulation of miRs in body fluids such as CSF, serum and urine, either by cell destruction or secretion. Therefore, miRs are attractive targets in the search of novel biomarkers $[10,11]$. In view of the complexity of miR sources, how to detect miRs that representing $\mathrm{AD}$ pathological changes has become one of the solutions to reduce false negatives in diagnosis.

In a previous study, we reported that miR-29c-3p was down-regulated in hippocampal neurons of APP/PS1 double transgenic mice, at the same time, the total miR-29c-3p in CSF increased significantly, suggesting that the reduced miR-29c-3p in neuronal cells may be released into CSF. However, the total miR-29c-3p in mouse plasma did not change significantly, and the plasma exosomal miR-29c-3p increased significantly, compared with the wild-type mice [12]. The decrease of MiR-29c-3p level may lead to the progression of AD [13]. These results suggested that miR-29c-3p in CSF was transported to peripheral blood through 
exosomes across the BBB. Further exosome protein mass spectrometry results showed that expression of the amphiphysin 1 in the CSF of AD patients was significantly higher than that of controls, suggested that amphiphysin 1 may be related to the increase of peripheral blood exosomal miR-29c-3p.

Previous multi-center studies have shown that the concentration of neural-derived exosomal A 342 , T-tau, and P-T181-tau in peripheral blood can reflect their changes in CSF [14]. In the present study, the neural cell adhesion molecule (NCAM) single-labeled exosomes were first captured, and then specific NCAM/amphiphysin 1 dual-labeled exosomal components are screened for corresponding detection. All subjects were tested for NCAM single-labeled and NCAM/amphiphysin 1 dual-labeled exosomal A $422, A \beta_{42 / 40}$, T-tau, P-T181-tau, NfL and miR-29c-3p. The results from discovery stage were validated a in a validation stage with more samples in different centers.

\section{Methods And Materials}

\subsection{Participants}

A total of 392 subjects were randomly selected from the Beijing Anding Hospital (center 1), Xuanwu Hospital of Capital Medical University (center 2), Minhang Hospital, Fudan University (center 3), Air Force General Hospital, Chinese People's Liberation (center 4), from May 2018 to March 2021 ; of which 92 subjects were in the SCD group, 95 subjects were in the $\mathrm{MCl}$ group, and 85 subjects were in the DAT group. Another 60 randomly selected healthy subjects were recruited as the control group. For the evaluation of the differential diagnosis, 60 patients with vascular dementia were also included. The diagnosis of $A D$ subject was made according to the criteria of the National Institute on Aging and Alzheimer's Association (NIA-AA) [15]. The diagnosis of aMCl subject was based on published criteria [16]. The diagnosis of SCD subject made according to published criteria and researches [17, 18]. The diagnosis of SCD subject made according to published criteria $[19,20]$. The experimental protocol was approved by the Ethics Committees of participating centers. Written informed consents were obtained from all participants or their legal guardians.

According to the patients enrolled in three central hospitals in Beijing as the discovery stage of the study, the patients enrolled in the two central hospitals in Shanghai and Heilongjiang province as the verification stage. Subjects with endocrine system, liver, kidney or cardiovascular diseases would be excluded. All samples were stored and detected in a blinded manner.

\subsection{Sample collection and pretreatment}

The EDTA anticoagulated venous blood was drawn in the morning after $8 \sim 12$ hours fasting (Vacutainer $\mathrm{K}_{2}$-EDTA tube, BD, Franklin Lake, USA). To reduce the error caused by the exchange of exosomes and blood, centrifugation is required within 30 minutes after specimen collection. Subjects undergoing blood cell exosomes release experiments were collected two tubes of specimens, and other subjects were collected one tube of specimens. To obtain plasma, the specimens were centrifuged at $4,200 \mathrm{~g}$ for 10 minutes at $4^{\circ} \mathrm{C}$; to obtain blood cells, blood separation medium was used according to the manufacturer's protocol (Solarbio, Beijing, China) [14]. The plasma was stored in liquid nitrogen before testing and was only allowed to freeze and thaw once. The blood cells were used in the next experiment within 1 hour.

The CSF samples were collected within 1 hour after blood samples collection in accordance to the CSF collection and biobanking guideline [21]. In brief, the patient was positioned in a left lateral position, and the puncture point is between the waist 3 and 4 . Each subject collected $5 \mathrm{ml}$ to $15 \mathrm{ml}$ of CSF. The collected $\mathrm{CSF}$ was centrifuged at $2,000 \mathrm{~g}$ at $4^{\circ} \mathrm{C}$ for $10 \mathrm{~min}[13,14]$. The supernatant was immediately collected into polypropylene tube, stored in liquid nitrogen before testing, and allowed to freeze and thaw only once.

\subsection{Blood cell separation and culture}

The separated RBCs and WBCs were washed in $37^{\circ} \mathrm{C}$ pre-warmed phosphate buffered saline (PBS) 3 times and then cultured in Roswell Park Memorial Institute 1640 (RPMI 1640) medium (Invitrogen, Carlsbad, USA) containing 10 \% exosome-depleted serum (Gibco, Thermo Fisher Scientific, MA, USA) at a concentration of $1 \times 10^{7} / \mathrm{L}$ for $0 \mathrm{~h}$ and $2 \mathrm{~h}$. The NCAM in the cells and medium were detected by enzyme linked immunosorbent assay (ELISA) method (Abcam, Cambridge, UK) according to the following procedure. The randomly selected and gender/age-matched SCD, aMCI, DAT, VaD and control samples from XuanWu Hospital were all run in triplicate.

\subsection{Neuronal-derived exosomes collection from plasma}

First of all, the total exosome was extracted from plasma. In brief, $0.5 \mathrm{ml}$ plasma was incubated with $0.15 \mathrm{ml}$ thromboplastin-D (Amresco, Boise, USA) for 60 min at room temperature, then $0.33 \mathrm{ml}$ magnesium and calcium-free Dulbecco's phosphate-buffered saline (D-PBS, Amresco), $0.02 \mathrm{ml}$ EDTA-free protease and phosphatase inhibitor cocktails (Abcam) were added. Next, $0.5 \mathrm{ml}$ of the mixture was added with $0.5 \mathrm{ml}$ of D-PBS, and the mixed solution was centrifuged at $1,500 \mathrm{~g}$ for 20 minutes at $4^{\circ} \mathrm{C}$. The supernatant was mixed with equal volume of D-PBS, and the mixed solution was centrifuged at $1,500 \mathrm{~g}$ for $20 \mathrm{minutes}$.

Then, specific neuronal-derived exosomes defined by multiple studies were separated by a novel immunomagnetic bead method [14, 22]. In short, $0.2 \mathrm{ml}$ of supernatant of previous step was mixed with $0.05 \mathrm{ml}$ of ExoQuick ${ }^{\mathrm{T}}$ exosome precipitation solution (System Biosciences, CA, USA) and incubated on ice for 1 hour. After centrifugation at 1,500 g for 30 minutes, the pellet was resuspended in $200 \mu \mathrm{L}$-PBS. $200 \mu \mathrm{l}$ of each sample was incubated with the NCAM monoclonal antibody (mouse anti-human, Abcam) coated Dynabeads ( $1 \mu \mathrm{m}$, Thermo Fisher Scientific) for 30 min in microplate on a shaker at $37^{\circ} \mathrm{C}$. Then use a magnet with a shape that fits the microplate to draw on the magnetic beads and discard the supernatant. Remove the magnet, each sample was resuspended in $210 \mu \mathrm{l} 0.05 \mathrm{M}$ glycine- $\mathrm{HCl}(\mathrm{pH} 3.0$, Amresco) mixed with $0.10 \%$ Tween 20 and $10 \mu$ inhibitor cocktails by shaking for 30 seconds and draw the beads with the magnet again. Then the supernatant was harvested and the $\mathrm{pH}$ of the supernatant was adjusted to $7.0 \mathrm{with} 1 \mathrm{M} \mathrm{Tris-} \mathrm{HCl}$ ( $\mathrm{pH} 8.6$, $\mathrm{Amresco}$ ).

Afterwards, specific subcomponent of neurogenic exosomes was captured. In brief, $200 \mu$ l of the supernatant from the previous step was added into the plate of a human amphiphysin 1 ELISA kit (Abcam). After 30 minutes incubation at $37^{\circ} \mathrm{C}$, the plate was washed three times with the wash solution provided in the 
kit and then drained. Specific exosomes were adsorbed on the well wall for the next step. The extraction reagent is directly added to the well to extract protein or nucleic acid for subsequent detection, according to the following procedure (Fig. 1).

\subsection{Transmission electron microscopy}

Exosomes adsorbed on the well wall were eluted with glycine- $\mathrm{HCl}(\mathrm{pH} 3.0)$ and adjusted $\mathrm{pH}$ to 7.0 with Tris- $\mathrm{HCl}(\mathrm{pH} 8.6)$, then the transmission electron microscopy (TEM) was performed according to published protocol [23]. Sections (65 nm) were stained with uranyl acetate and Reynold's lead citrate. The experiment was performed on JEM-2100F TEM (JEOL, Akishima-shi, Japan).

\subsection{MiR purification and miR analysis}

Total miR was purified using a magnetic bead method Kit (Thermo Fisher Scientific) according to the manufacturer's protocol. Total RNA yields were $\sim 20$ $\mathrm{ng} / \mathrm{mL}$ and $\sim 50 \mathrm{ng} / \mathrm{mL}$ in CSF and plasma respectively, as assessed by Quant-iT ${ }^{\mathrm{m}}$ RiboGreen ${ }^{\mathrm{Tw}}$ RNA reagent (Invitrogen). Total miR in blood cells was extracted by a column-based method Kit (Thermo Fisher Scientific). MiRs were reverse transcribed into cDNA with $10 \mu$ l reaction volume, followed by TaqMan qPCR according to the manufacturer's protocol (Thermo Fisher Scientific). Briefly, a $20 \mu \mathrm{l} \mathrm{PCR}$ reaction contained $2 \mu \mathrm{l}$ cDNA, $300 \mathrm{nM}$ TaqMan probe, $300 \mathrm{nM}$ sense primer, and $300 \mathrm{nM}$ anti-sense primer. Cycling parameters were $95^{\circ} \mathrm{C}$ for $8 \mathrm{~min}$, followed by 35 cycles of $95^{\circ} \mathrm{C}$ for $15 \mathrm{~s}$ and $60^{\circ} \mathrm{C}$ for $1 \mathrm{~min}$ (Roche Light Cycler 480, Basel, Switzerland). For body fluid samples, miRNeasy Serum/Plasma Spike-In Control (C. elegans miR-39 miR mimic, Qiagen, Dusseldorf, Germany) served as control; while for cell samples, U6 snRNA (RNU6B, Qiagen) served as endogenous control. The relative levels of miRs were calculated by the $2^{-\Delta \Delta C t}$ method [24].

\subsection{Western blot}

Western blot was performed to detect exosomal biomarker, Alix, as well as NCAM and amphiphysin 1 using monoclonal anti-human antibodies (Santa Cruz, Dallas, USA). The dilution ratios of the primary and secondary antibodies of Alix, NCAM and amphiphysin 1 were 1:1000/1:400, 1:500/1:300, 1:1000/1:300 respectively. The captured exosomes and the corresponding supernatant in all steps were tested for the above three proteins.

\subsection{ELISA measurements of protein}

The A 42 , A 440 , T-tau, P-T181-tau, and NfL (Abcam) ELISA tests were performed in strict accordance with the instructions of the kit. The determination of CD81 content is used for the normalization of exosomes content. The mean value of CD81 levels in each group was set to 1.00 , and the relative values for each sample were used to normalize their recovery [14].

\subsection{Statistical analyses}

Statistical analyses were performed using SPSS 18.0 for Windows (SPSS, Inc., Chicago, IL, USA). For normally distributed data, results are expressed as the mean \pm standard deviation. The differences between groups were assessed using the t-test or one-way ANOVA. Rates were compared using the chi-square $\left(\chi^{2}\right)$ test. Correlations were determined by computing the Spearman Rank Correlation. The Delong test was used to compare the performance of two ROC curves. A binary logistic regression model was used to calculate the predicted values, where age, gender, and APOE status were used as covariates. $P<0.05$ was considered to indicate a statistically significant difference.

\section{Results}

\subsection{Participant characteristics}

Table 1 depicts the characteristics of participants. There were no differences in the ages or ratios of males/females among the SCD, aMCI, AD, VaD and control groups in both the discovery stage and the validation stage. The percentages of APOE $\varepsilon 4$, Mini-Mental State Examination (MMSE), and Clinical Dementia Rating (CDR) scores were significantly different between aMCl patients and controls, $A D$ patients and controls, $V a D$ patients and controls, and $A D$ and aMCl patients (all $P<0.05$ ). 
Table 1

Demographics and clinical characteristics of subjects in this study

\begin{tabular}{|c|c|c|c|c|c|c|c|c|c|c|}
\hline \multirow[t]{2}{*}{ Characteristic } & \multirow[t]{2}{*}{ Total } & \multicolumn{5}{|c|}{ Discovery Stage $(n=195)$} & \multicolumn{4}{|c|}{ Verification Stage $(n=197)$} \\
\hline & & Control & $\mathrm{VaD}$ & SCD & $\mathrm{aMCl}$ & $A D$ & Control & $\mathrm{VaD}$ & SCD & $\mathrm{aMc}$ \\
\hline $\begin{array}{l}\text { No. of } \\
\text { subjects }\end{array}$ & 392 & 30 & 30 & 45 & 50 & 40 & 30 & 30 & 47 & 45 \\
\hline $\begin{array}{l}\text { Age, mean } \\
(\mathrm{SD}) \text {, years }\end{array}$ & $65.9(6.6)$ & $\begin{array}{l}65.2 \\
(6.5)\end{array}$ & $65.7(6.1)$ & $\begin{array}{l}66.1 \\
(5.9)\end{array}$ & $65.5(7.1)$ & $66.3(6.5)$ & $\begin{array}{l}65.3 \\
(6.1)\end{array}$ & $66.5(5.6)$ & $\begin{array}{l}65.8 \\
(5.5)\end{array}$ & 66.1 \\
\hline Males, No. (\%) & $190(48.5)$ & $15(50.0)$ & $15(50.0)$ & $20(44.4)$ & $25(50.0)$ & $20(50.0)$ & $15(50.0)$ & $15(50.0)$ & $21(44.7)$ & 22( \\
\hline $\begin{array}{l}\text { Education } \\
\text { years, mean } \\
\text { (SD) }\end{array}$ & $9.3(1.5)$ & $9.3(1.4)$ & $9.9(1.5)$ & $9.1(1.5)$ & $9.5(1.4)$ & $9.9(1.4)$ & $9.3(1.4)$ & $9.3(1.5)$ & $9.5(1.4)$ & 9.21 \\
\hline $\begin{array}{l}\text { Body Mass } \\
\text { Index, mean } \\
\text { (SD) }\end{array}$ & $25.9(2.1)$ & $\begin{array}{l}25.3 \\
(1.8)\end{array}$ & $25.9(1.9)$ & $\begin{array}{l}26.2 \\
(2.1)\end{array}$ & $25.8(2.3)$ & $26.0(2.2)$ & $\begin{array}{l}25.6 \\
(2.1)\end{array}$ & $26.3(2.1)$ & $\begin{array}{l}25.7 \\
(1.5)\end{array}$ & $26 . C$ \\
\hline $\begin{array}{l}\text { ApoE } \varepsilon 4 \\
\text { positive, No. } \\
(\%)\end{array}$ & $58(14.8)$ & $6(20.0)$ & $5(16.7)$ & $6(13.3)$ & $5(10.0)$ & $7(17.5)$ & $5(16.7)$ & $3(10.0)$ & $8(17.0)^{\star}$ & $6(1:$ \\
\hline $\begin{array}{l}\text { MMSE score, } \\
\text { mean (SD) }\end{array}$ & $25.0(1.9)$ & $\begin{array}{l}29.5 \\
(1.3)\end{array}$ & $\begin{array}{l}25.2 \\
(0.6)^{*}\end{array}$ & $\begin{array}{l}28.6 \\
(1.1)\end{array}$ & $26.1(0.6)^{\star \dagger}$ & $20.9(2.9)^{\star} \S$ & $\begin{array}{l}29.7 \\
(1.4)\end{array}$ & $\begin{array}{l}24.9 \\
(0.9)^{*}\end{array}$ & $\begin{array}{l}28.5 \\
(1.0)\end{array}$ & 25.8 \\
\hline $\begin{array}{l}\text { CDR score of } \\
0 / 0.5 / 1.0 / 2.0, \\
\text { No. }\end{array}$ & $206 / 118 / 46 / 22$ & $29 / 1 / 0 / 0$ & $9 / 19 / 2 / 0^{*}$ & $43 / 2 / 0 / 0$ & $22 / 28 / 0 / 0^{\star+}$ & $0 / 11 / 19 / 10^{\star} \S$ & $30 / 0 / 0 / 0$ & $8 / 17 / 5 / 0^{*}$ & $44 / 3 / 0 / 0$ & $21 /:$ \\
\hline $\begin{array}{l}\text { Homocysteine, } \\
\text { mean (SD), } \\
\mu \mathrm{mol} / \mathrm{L}\end{array}$ & $13.5(3.5)$ & $8.2(2.1)$ & $\begin{array}{l}10.5 \\
(3.6)^{*}\end{array}$ & $\begin{array}{l}16.7 \\
(4.8)^{*}\end{array}$ & $15.9(3.9)^{\star+}$ & $16.8(3.2)^{\star} \S$ & $9.2(2.3)$ & $\begin{array}{l}11.8 \\
(3.0)^{*}\end{array}$ & $\begin{array}{l}16.5 \\
(3.9)^{*}\end{array}$ & 17.3 \\
\hline $\begin{array}{l}\text { Hypertension } \\
\text { treatment, No. } \\
(\%)\end{array}$ & 64 (16.3) & 5 (16.7) & $6(20.0)$ & 7 (15.6) & 7 (14.0) & 5 (12.5) & $6(20.0)$ & 5 (16.7) & 7 (14.9) & $8(1$ \\
\hline $\begin{array}{l}\text { Statin } \\
\text { treatment, No. }\end{array}$ & $86(21.9)$ & $6(20.0)$ & $15(50.0)^{*}$ & $5(11.1)$ & $8(16.0)$ & 7 (17.5) & $5(16.7)$ & $21(70.0)^{*}$ & $8(17.0)$ & $6(1:$ \\
\hline
\end{tabular}

$(\%)$

Note: $\operatorname{APOE} \varepsilon 4$, apolipoprotein $\varepsilon 4$; MMSE, Mini-Mental State Examination; CDR, clinical dementia rating.

* $P<0.05$ compared to controls; $\uparrow P<0.05$ compared to SCD; $\S P<0.05$ compared to aMCI.

\subsection{Identification of exosomes}

The results of TEM and particle size analysis show that the shape and size of the extract were consistent with the characteristics of exosomes (Fig. 2A, B). Secondly, western blot results show that Alix and L1CAM in the stage 1 immune capture product were highly expressed but not detected in supernatants (Fig. 2C, D). Third, the results of ELISA showed that in the stage 1 and stage 2 immune capture, the levels of NCAM and amphiphysin 1 in the captured product were higher than that of the corresponding supernatant respectively (all $P<0.05$ ) (Fig. 2E, F).

The concentration of exosomes in all specimens were standardized using CD81 test results before testing for other proteins. Overall, there was no significant difference in CD81 among the groups (all $P<0.05$ ) (Fig. 2G).

More important, after 2 hours of cultivation, NCAM levels in WBCs and RBCs did not decrease significantly (all $P>0.05$ ), the NCAM levels in WBC and RBC medium did not increase significantly (all $P>0.05$ ) (Fig. 3). The result suggested that the content of NCAM in RBCs and WBCs were low ( $30 \mu \mathrm{g} / \mathrm{L}$ ), RBC and WBC almost did not release NCAM into the medium, suggested that specimen storage and pre-processing will not affect subsequent test results.

\subsection{Levels of $A \beta 42, A \beta_{42 / 40}$, T-tau, P-T181-tau, NfL, and miR-29c-3p in NCAM single- labeled exosomes of plasma}

We first use the specimens from the discovery stage to detect the NCAM-labeled exosomal proteins extracted in the first stage of the exosome extraction experiment. Exosomes of this component are generally considered neuron-derived exosomes. The results showed that the levels of $A \beta 42, A \beta_{42 / 40}$, Tau and $P$ T181-tau in plasma exosomes of the $\mathrm{aMCl}$ and $\mathrm{AD}$ groups were significantly higher than those of the SCD group, control group and VaD group, and the AD group was higher than the aMCl group. The levels of plasma exosomal A 42 , Tau and P-T181-tau in the SCD group were slightly higher than those in the control group and the $\mathrm{VaD}$ group, but there was no significant difference. The level of $\mathrm{NfL}$ in plasma exosomes in the aMCl and $\mathrm{AD}$ groups was significantly higher than that in the SCD group, control group and VaD group, and the AD group was higher than that in the aMCl group. The NfL level of plasma exosomes in the SCD group was slightly higher than that in the control group and the VaD group, but there was no significant difference. The levels of miR-29c-3p in plasma exosomes in the $\mathrm{aMCl}$ and $\mathrm{AD}$ groups were significantly higher than that in the $\mathrm{SCD}$, control, and VaD group; while the AD group was higher than the aMCl group. The level of plasma exosomes miR-29c-3p in the SCD group was slightly higher than that in the control group and the VaD group, but there was no significant difference. The change trend of the test results of the specimens in the verification stage is consistent with the discovery stage (Fig. 4). 
We first used the specimens from the discovery stage to detect the NCAM/amphiphysin 1 dual-labeled exosomal proteins extracted in the second stage of the exosome extraction experiment. The results showed that the levels of $A \beta_{42 / 40}$, Tau, and P-T181-tau in plasma exosomes of the aMCl and $A D$ groups were significantly higher than those of the SCD group, control group and $\mathrm{VaD}$ group, and the AD group was higher than the aMCl group. The levels of plasma exosomes $A \beta_{42 / 40}$, Tau, and P-T181-tau in the SCD group were slightly higher than those in the control group and the VaD group, but there was no significant difference. There was no significant difference in the level of exosomal A 340 among the groups, and only the AD group decreased slightly. The level of NfL in plasma exosomes in the $\mathrm{aMCl}$ and $\mathrm{AD}$ groups was significantly higher than that in the SCD group, control group and VaD group, and the $\mathrm{AD}$ group was higher than that in the aMCl group. The NfL level of plasma exosomes in the SCD group was slightly higher than that in the control group and the VaD group, but there was no significant difference. The change trend of the test results of the specimens in the verification stage was consistent with the discovery stage. In all disease groups and control groups, the levels of $A \beta 42$ and $A \beta_{42 / 40}$ in NCAM/amphiphysin 1 dual-labeled exosomes were higher than those of NCAM singlelabeled exosomes, considering that the concentration of exosomes in each group has been standardized by CD81.

The levels of Aß42 and miR-29c-3p in plasma NCAM/amphiphysin 1 dual-labeled exosomes of the SCD, aMCl and AD groups were significantly higher than those of the control and $\mathrm{VaD}$ groups, the $\mathrm{AD}$ group was higher than the $\mathrm{aMCl}$ group, and the aMCl group was higher than the $\mathrm{SCD}$ group (Fig. 5).

Of interest, the increase ratio of NCAM/amphiphysin 1 double-labeled exosomes AB42 and miR-29c-3p in all disease groups was higher than that of T-tau, PT181-tau, and NfL (Fig. 6).

\subsection{Correlation analysis between CSF and plasma exosomal biomarkers}

We conducted a correlation analysis of exosomes and CSF biomarkers and found that the levels of AB42, P-T181-tau, as well as miR-29c-3p in neuron-derived exosomes were highly correlated with their levels in CSF in all groups (all $P<0.05$ ), while the level of $\mathrm{NfL}$ in neuron-derived exosomes has no correlation with CSF (all $P>0.05$ ). This correlation was also observed in NCAM/amphiphysin 1 dual-labeled exosomes, and the correlation coefficients were even higher (Fig. 7).

\subsection{Diagnostic power analysis of plasma exosomal biomarkers}

We used the ROC curve to analyze the diagnostic performance of A 42 , A $\beta_{42 / 40}$, T-tau, P-T181-tau, NfL, and miR-29c-3p in plasma NCAM single-labeled (Fig. 8, Table 2) and NCAM/amphiphysin 1 dual-labeled (Fig. 9, Table 3) exosome. Plasma NCAM/amphiphysin 1 dual-labeled exosomal A $\beta_{42 / 40}$ and miR-29c-3p showed a good diagnostic performance. For $\mathrm{A} \beta_{42 / 40}$, the AUCs for the SCD/controls, aMCl/controls and $\mathrm{AD} /$ controls comparisons were $0.698(P=0.000)$ and $0.884(P=.000), 0.969(P=0.000)$, respectively; the AUCs for the aMCl/SCD and AD/aMCl were $0.828(P=0.000)$ and $0.806(P=0.000)$, respectively. For miR29c-3p, the AUCs for the SCD/controls, $\mathrm{AD} /$ controls and aMCl/controls comparisons were $0.876(P=0.000)$ and $0.906(P=0.000), 0.915(P=0.000)$, respectively; the AUCs for the SCD/aMCl and aMCl/AD were $0.778(P=0.000)$ and $0.670(P=0.000)$, respectively. The results of DeLong test showed that the AUC of NCAM/amphiphysin 1 dual-labeled exosomal A $\beta_{42 / 40}$ for diagnosis of SCD was higher than that of A $342(P=0.032)$, T-tau $(P=0.001)$ and P-T181-tau $(P=0.025)$; the AUC of NCAM/amphiphysin 1 dual-labeled exosomal miR-29c-3p for diagnosis of SCD was higher than that of $\mathrm{A} \beta 42(P=0.027)$, A $\beta_{42 / 40}(P=$ 0.001), T-tau $(P=0.001)$, P-T181-tau $(P=0.008)$, and NfL $(P=0.000)$. These data indicate that plasma NCAM/amphiphysin 1 dual-labeled exosomal A $\beta_{42 / 40}$ and miR-29c-3p had potential advantages in the diagnosis of SCD.

Table 2

The ROC curve analyze results of NCAM single labeled exosomal biomarkers.

\begin{tabular}{|c|c|c|c|c|c|c|c|c|c|c|c|c|c|c|c|}
\hline & \multirow[t]{2}{*}{ Stage } & \multicolumn{2}{|c|}{ VaD vs. Con } & \multicolumn{2}{|c|}{ SCD vs. Con } & \multicolumn{2}{|c|}{ aMCl vs. Con } & \multicolumn{2}{|c|}{$A D$ vs. Con } & \multicolumn{2}{|c|}{ SCD vs. VaD } & \multicolumn{2}{|c|}{ aMCl vs. SCD } & \multicolumn{2}{|c|}{$\mathrm{AD}$ vs. aMCl } \\
\hline & & AUC & $P$ & AUC & $P$ & AUC & $P$ & AUC & $P$ & AUC & $P$ & AUC & $P$ & AUC & $P$ \\
\hline \multirow[t]{2}{*}{ Aß42 } & Discovery & 0.557 & 0.285 & 0.651 & 0.004 & 0.834 & 0.000 & 0.879 & 0.000 & 0.618 & 0.026 & 0.734 & 0.000 & 0.617 & 0.027 \\
\hline & Verification & 0.504 & 0.939 & 0.641 & 0.051 & 0.841 & 0.000 & 0.890 & 0.000 & 0.657 & 0.003 & 0.747 & 0.000 & 0.637 & 0.051 \\
\hline \multirow[t]{2}{*}{$A \beta_{42 / 40}$} & Discovery & 0.534 & 0.517 & 0.571 & 0.181 & 0.802 & 0.000 & 0.887 & 0.000 & 0.532 & 0.541 & 0.754 & 0.000 & 0.666 & 0.002 \\
\hline & Verification & 0.529 & 0.583 & 0.537 & 0.482 & 0.802 & 0.000 & 0.891 & 0.000 & 0.505 & 0.919 & 0.783 & 0.000 & 0.678 & 0.001 \\
\hline \multirow[t]{2}{*}{ T-tau } & Discovery & 0.573 & 0.168 & 0.566 & 0.216 & 0.859 & 0.000 & 0.885 & 0.000 & 0.514 & 0.797 & 0.833 & 0.000 & 0.558 & 0.270 \\
\hline & Verification & 0.565 & 0.217 & 0.558 & 0.276 & 0.876 & 0.000 & 0.908 & 0.000 & 0.509 & 0.862 & 0.852 & 0.000 & 0.576 & 0.149 \\
\hline \multirow[t]{2}{*}{ P-T181-tau } & Discovery & 0.500 & 0.994 & 0.502 & 0.996 & 0.857 & 0.000 & 0.878 & 0.000 & 0.503 & 0.963 & 0.846 & 0.000 & 0.546 & 0.389 \\
\hline & Verification & 0.541 & 0.440 & 0.533 & 0.537 & 0.879 & 0.000 & 0.879 & 0.000 & 0.509 & 0.858 & 0.868 & 0.000 & 0.531 & 0.564 \\
\hline \multirow[t]{2}{*}{$\mathrm{NfL}$} & Discovery & 0.501 & 0.985 & 0.508 & 0.875 & 0.676 & 0.001 & 0.751 & 0.000 & 0.513 & 0.813 & 0.693 & 0.000 & 0.586 & 0.103 \\
\hline & Verification & 0.522 & 0.673 & 0.508 & 0.879 & 0.742 & 0.000 & 0.803 & 0.000 & 0.529 & 0.582 & 0.746 & 0.000 & 0.599 & 0.062 \\
\hline \multirow[t]{2}{*}{ miR-29c-3p } & Discovery & 0.527 & 0.616 & 0.740 & 0.000 & 0.846 & 0.000 & 0.884 & 0.000 & 0.726 & 0.000 & 0.663 & 0.002 & 0.712 & 0.000 \\
\hline & Verification & 0.528 & 0.598 & 0.717 & 0.000 & 0.853 & 0.000 & 0.808 & 0.000 & 0.738 & 0.000 & 0.661 & 0.002 & 0.669 & 0.001 \\
\hline
\end{tabular}


Table 3

The ROC curve analyze results of NCAM/amphiphysin 1 dual labeled exosomal biomarkers.

\begin{tabular}{|c|c|c|c|c|c|c|c|c|c|c|c|c|c|c|c|}
\hline & \multirow[t]{2}{*}{ Stage } & \multicolumn{2}{|c|}{ VaD vs. Con } & \multicolumn{2}{|c|}{ SCD vs. Con } & \multicolumn{2}{|c|}{ aMCl vs. Con } & \multicolumn{2}{|c|}{ AD vs. Con } & \multicolumn{2}{|c|}{ SCD vs. VaD } & \multicolumn{2}{|c|}{ aMCI vs. SCD } & \multicolumn{2}{|c|}{$\mathrm{AD}$ vs. aMCl } \\
\hline & & AUC & $P$ & AUC & $P$ & AUC & $P$ & AUC & $P$ & AUC & $P$ & AUC & $P$ & AUC & $P$ \\
\hline \multirow[t]{2}{*}{$A \beta 42$} & Discovery & 0.538 & 0.477 & 0.500 & 0.999 & 0.749 & 0.000 & 0.910 & 0.000 & 0.538 & 0.472 & 0.735 & 0.000 & 0.802 & 0.000 \\
\hline & Verification & 0.549 & 0.353 & 0.590 & 0.090 & 0.653 & 0.004 & 0.904 & 0.000 & 0.644 & 0.006 & 0.735 & 0.000 & 0.811 & 0.000 \\
\hline \multirow[t]{2}{*}{$A \beta_{42 / 40}$} & Discovery & 0.506 & 0.916 & 0.698 & 0.000 & 0.884 & 0.000 & 0.969 & 0.000 & 0.715 & 0.000 & 0.828 & 0.000 & 0.806 & 0.000 \\
\hline & Verification & 0.533 & 0.537 & 0.671 & 0.001 & 0.885 & 0.000 & 0.973 & 0.000 & 0.717 & 0.000 & 0.832 & 0.000 & 0.784 & 0.000 \\
\hline \multirow[t]{2}{*}{ T-tau } & Discovery & 0.503 & 0.962 & 0.598 & 0.064 & 0.856 & 0.000 & 0.910 & 0.000 & 0.591 & 0.087 & 0.796 & 0.000 & 0.703 & 0.000 \\
\hline & Verification & 0.536 & 0.493 & 0.588 & 0.095 & 0.861 & 0.000 & 0.908 & 0.000 & 0.566 & 0.214 & 0.806 & 0.000 & 0.691 & 0.000 \\
\hline \multirow[t]{2}{*}{ P-T181-tau } & Discovery & 0.540 & 0.445 & 0.591 & 0.084 & 0.849 & 0.000 & 0.900 & 0.000 & 0.553 & 0.319 & 0.807 & 0.000 & 0.662 & 0.002 \\
\hline & Verification & 0.511 & 0.842 & 0.568 & 0.198 & 0.834 & 0.000 & 0.898 & 0.000 & 0.576 & 0.153 & 0.785 & 0.000 & 0.668 & 0.002 \\
\hline \multirow[t]{2}{*}{$\mathrm{NfL}$} & Discovery & 0.501 & 0.979 & 0.502 & 0.971 & 0.706 & 0.000 & 0.838 & 0.000 & 0.503 & 0.950 & 0.787 & 0.000 & 0.622 & 0.021 \\
\hline & Verification & 0.521 & 0.698 & 0.537 & 0.490 & 0.696 & 0.000 & 0.843 & 0.000 & 0.506 & 0.904 & 0.757 & 0.000 & 0.578 & 0.139 \\
\hline \multirow[t]{2}{*}{ miR-29c-3p } & Discovery & 0.502 & 0.973 & 0.862 & 0.000 & 0.915 & 0.000 & 0.906 & 0.000 & 0.804 & 0.000 & 0.778 & 0.000 & 0.670 & 0.001 \\
\hline & Verification & 0.517 & 0.743 & 0.876 & 0.000 & 0.914 & 0.000 & 0.909 & 0.000 & 0.802 & 0.000 & 0.829 & 0.000 & 0.768 & 0.000 \\
\hline
\end{tabular}

Table 4

The ROC curve analyze results of combination biomarkers.

\begin{tabular}{|c|c|c|c|c|c|c|c|c|c|c|c|c|c|c|}
\hline \multirow[t]{2}{*}{ Composite } & \multicolumn{2}{|c|}{ VaD vs. Con } & \multicolumn{2}{|c|}{ SCD vs. Con } & \multicolumn{2}{|c|}{ aMCl vs. Con } & \multicolumn{2}{|c|}{ AD vs. Con } & \multicolumn{2}{|c|}{ SCD vs. VaD } & \multicolumn{2}{|c|}{ aMCl vs. SCD } & \multicolumn{2}{|c|}{$\mathrm{AD}$ vs. aMCl } \\
\hline & AUC & $P$ & AUC & $P$ & AUC & $P$ & AUC & $P$ & AUC & $P$ & AUC & $P$ & AUC & $P$ \\
\hline Composite-1 & 0.504 & 0.939 & 0.718 & 0.000 & 0.855 & 0.000 & 0.919 & 0.000 & 0.730 & 0.000 & 0.800 & 0.000 & 0.832 & 0.000 \\
\hline Composite-2 & 0.539 & 0.466 & 0.810 & 0.000 & 0.879 & 0.000 & 0.915 & 0.000 & 0.854 & 0.000 & 0.711 & 0.000 & 0.878 & 0.000 \\
\hline Composite-3 & 0.557 & 0.285 & 0.823 & 0.000 & 0.908 & 0.000 & 0.927 & 0.000 & 0.821 & 0.000 & 0.814 & 0.000 & 0.919 & 0.000 \\
\hline
\end{tabular}

\subsection{Analysis of diagnostic efficacy of combination biomarkers}

Further, we used logistic regression analysis and ROC to evaluate the diagnostic efficacy of the combination of the above-mentioned biomarkers for $A D$ (Fig. 10). The performances of biomarkers in plasma NCAM single-labeled exosomes, plasma NCAM/amphiphysin 1 dual-labeled exosomes or CSF as composite-1, composite-2, and composite-3 respectively. We compared the AUCs between the discovery and validation data sets and found no differences in the AUCs of each biomarker, so the data of the two stages was integrated for analysis. The three combinations achieved similar diagnostic performance. Furthermore, these data indicated that the combination of exosomal biomarkers had slightly higher diagnostic efficiency than the individual biomarkers and that the exosomal biomarkers had the same diagnostic power as the CSF biomarkers.

\section{Discussion}

Exosomes are tiny vesicles actively secreted by cells, which carry materials and biological information transmitted by cells [25]. In recent years, a variety of specific exosome capture techniques have been developed, such as microfluidic chip technology and immune capture [14, 22]. The core principle of the immune capture method is based on the size of the exosomes (30-200 nm), which is comparable to the size of virus-like particles and viruses (within 300 $\mathrm{nm}$ ) that can be captured by antibodies [26]. On the basis of the previous research, this study combined the magnetic bead method and the microtiter plate method to make it possible to harvest the exosomes with dual-specific biomarkers, and obtained ideal AD diagnostic performance.

In the year 2015, a single-center study reported that peripheral blood exosomal A 342 , T-tau, and P-T181-tau can identify or predict AD up to 10 years before clinical onset [22]. The characteristics of exosomal AB42, T-tau, and P-T181-tau were different from NfL, which was mainly used for diagnosis and early warning of familial AD [8]. Further more, the results of a multi-center study confirmed the above conclusion. The peripheral blood and CSF test results of 298 subjects from 8 centers showed that the NCAM single-labeled A 42 and other indicators have a good correlation with CSF [14]. Considering that the level of $A \beta 42$ in CSF can reflect the degree of amyloid plate and other pathological changes in brain tissue, the level of $A \beta 42$ in plasma exosomes should reflect the degree of amyloid plate in brain tissue to a certain extent. Our findings further confirm that plasma NCAM single-labeled exosomal A $342, A \beta_{42 / 40}, T$-tau, and PT181-tau have promising applications in the diagnosis of $\mathrm{aMCl}$ and $\mathrm{AD}$ as well as the differential diagnosis of VaD, and might be used as a substitute for cerebrospinal fluid Potential markers for inspection and PET inspection.

BACE-1 is one of the key enzymes for the occurrence and development of AD. Our previous research results showed that miR-29c-3p could down-regulate the expression and activity of BACE-1 [12]. However, with the development of AD, the level of miR-29c-3p in neuronal cells continued to decrease. Further animal 
experiments and clinical studies have shown that with the progress of $A D$, the level of miR-29c-3p in cerebrospinal fluid and peripheral blood exosomes keep rising, which suggests that the decrease of miR-29c-3p in neuronal cells may be due to excessive miR-29c-3p being transported from neurons to cerebrospinal fluid and peripheral blood.

The pathogenesis of $A D$ is diverse. At present, the mainstream ones include amyloid theory, genetic theory, tau protein theory and lipid metabolism theory. In the theory of lipid metabolism, apolipoprotein E4 (ApoE4) occupies an important position, which plays a role in both amyloid production and tau protein phosphorylation [15]. Similar to ApoE4, amphiphysin 1 is widely distributed in various tissues and organs including brain tissue [27]. It can transport cholesterol and other lipids from the cell to the extracellular environment on the basis of consuming adenosine triphosphate, which is important in lipid metabolism. Studies have shown that amphiphysin 1 can interact with ApoE4 and act as a component of the amyloid clearance channel, so the change of amphiphysin 1 expression may be involved in the occurrence and development of AD [28]. The main modeling principle of APP/PS1 model mice is to promote the excessive deposition of amyloid. Therefore, our research group found that the significant changes in the expression of amphiphysin 1 in exosomes may be caused by abnormal amyloid metabolism. As a membrane protein, amphiphysin 1 also may be actively loaded on the exosomal membrane by the cell during the exosome production stage and secreted to the outside of the cell. There are multiple ways of $A \beta$ elimination, such as endocytosis, proteases degradation, lymphatic drainage, CSF flow and active transportation across the BBB. Soluble A $\beta$ also can be actively transported into BBB endothelial cells via amphiphysin 1, ApoE and LRP1 pathway, and be degraded in endothelial cells or pass through BBB to the peripheral blood [28, 29]. The neuronal parenchymal cells are redistributed by the amphiphysin 1 transporter, which is considered to be the prime modulator of cholesterol homeostasis in the brain.

Current study showed that the diagnostic efficacy of miR-29c-3p of peripheral blood NCAM single-labled exosomes for AD and aMCl is similar to that of $A \beta 42$, but their diagnostic value for SCD was limited. However, miR-29c-3p in NCAM/amphiphysin 1 dual-labeled exosomes of peripheral blood not only has better diagnostic performance for $\mathrm{AD}$ and $\mathrm{aMCl}$, but also has acceptable diagnostic performance for $\mathrm{SCD}$, which is not available in peripheral blood exosomes $\mathrm{A} \beta 42$.

In addition, for T-tau, P-T181-tau and NfL, there was no significant difference in the levels of NCAM single-labeled exosomes and NCAM/amphiphysin 1 double-labeled exosomes, as well as the diagnostic efficiency of AD diagnosis and staging, which suggested that there is no need to detect NCAM/amphiphysin 1 dual-labeled exosomal T-tau, P-T181-tau and NfL for diagnosis and staging of AD. These data indicate that blood neuron-derived exosomes are ideal biomarker carriers for AD screening, especially for large-scale population screening.

In summary, this study first provided a method for sorting specific surface marker exosomes using a two-step method; and verified that peripherald neuronalderived exosomal $A \beta 42, A \beta_{42 / 40}$, T-tau, P-T181-tau and miR-29c-3p may reflect AD pathological changes in the brain and therefore have the capacity to diagnose AD. The detection of NCAM/amphiphysin 1 dual-labeled exosomal molecules show a better potential diagnostic value for SCD than NCAM singlelabeled exosomes. However, these findings need further confirmation in longitudinal studies.

\section{Declarations}

\section{Ethics approval}

The experimental protocol was approved by the Ethics Committees of participating centers.

\section{Consent to participate}

Written informed consents were obtained from all participants or their legal guardians.

\section{Consent for publication}

Not applicable

\section{Authors' contributions}

Ying Li, Ming Xia, Shuang Meng, and Di Wu tested patient specimens and analyzed the data. Ying Li performed the cell culture and was a major contributor in writing the manuscript. Sihai Ling and Xiali Chen participated in data analysis. Chengeng Liu mainly participated in the experimental design. All authors read and approved the final manuscript.

\section{Conflicts of Interest}

The authors declare that they have no conflicts of interest.

\section{Funding}

This study was supported by the National Natural Science Foundation of China (No. 81401734), and the National Key Research and Development Program of China (2016YFC1306300, 2019YFF0216502).

\section{Data Availability}

The data used to support the findings of this study are available from the first author or corresponding author upon request.

\section{Acknowledgments}


We would like to thank Yang Zhi, Xiuyu Li, Yunxiu Zhang, Xiaoling Guan, Bei Li, Tingting Yang, Yu Liu, Lili Li, Fang Li, and He Lan for excellent assistance with sample collection and processing.

\section{References}

1. Hane FT, Robinson M, Lee BY, Bai O, Leonenko Z, Albert MS. Recent progress in Alzheimer's disease research, part 3: diagnosis and treatment. J Alzheimers Dis. 2017; 57(3): 645-665.

2. Molinuevo JL, Ayton S, Batrla R, Bednar MM, Bittner T, Cummings J, Fagan AM, Hampel H, Mielke MM, Mikulskis A, O'Bryant S, Scheltens P, Sevigny J, Shaw LM, Soares HD, Tong G, Trojanowski JQ, Zetterberg H, Blennow K. Current state of Alzheimer's fluid biomarkers. Acta Neuropathol. 2018; 136(6): 821-853.

3. Anderson ND. State of the science on mild cognitive impairment (MCl). CNS Spectr. 2019; 24(1): 78-87.

4. Jessen F, Amariglio RE, Buckley RF, van der Flier WM, Han Y, Molinuevo JL, Rabin L, Rentz DM, Rodriguez-Gomez O, Saykin AJ, Sikkes SAM, Smart CM, Wolfsgruber S, Wagner M. The characterisation of subjective cognitive decline. Lancet Neurol. 2020; 19(3): 271-278.

5. Okazawa H. Ultra-early phase pathologies of Alzheimer's disease and other neurodegenerative diseases. Proc Jpn Acad Ser B Phys Biol Sci. 2017; 93(6): 361-377.

6. Bao W, Xie F, Zuo C, Guan Y, Huang YH. PET neuroimaging of Alzheimer's disease: radiotracers and their utility in clinical research. Front Aging Neurosci. 2021; 13: 624330 .

7. Okazawa H. Intracellular amyloid hypothesis for ultra-early phase pathology of Alzheimer's disease. Neuropathology. 2021; 41(2): 93-98.

8. Preische O, Schultz SA, Apel A, Kuhle J, Kaeser SA, Barro C, Gräber S, Kuder-Buletta E, LaFougere C, Laske C, Vöglein J, Levin J, Masters CL, Martins R, Schofield PR, Rossor MN, Graff-Radford NR, Salloway S, Ghetti B, Ringman JM, Noble JM, Chhatwal J, Goate AM, Benzinger TLS, Morris JC, Bateman RJ, Wang G, Fagan AM, McDade EM, Gordon BA, Jucker M; Dominantly Inherited Alzheimer Network. Serum neurofilament dynamics predicts neurodegeneration and clinical progression in presymptomatic Alzheimer's disease. Nat Med. 2019 Feb; 25(2): $277-283$.

9. Hampel H, O'Bryant SE, Molinuevo JL, Zetterberg H, Masters CL, Lista S, Kiddle SJ, Batrla R, Blennow K. Blood-based biomarkers for Alzheimer disease: mapping the road to the clinic. Nat Rev Neurol. 2018; 14(11): 639-652.

10. Zetterberg H, Burnham SC. Blood-based molecular biomarkers for Alzheimer's disease. Mol Brain. 2019. 12(1): 26.

11. Nagaraj S, Zoltowska KM, Laskowska-Kaszub K, Wojda U. microRNA diagnostic panel for Alzheimer's disease and epigenetic trade-off between neurodegeneration and cancer. Ageing Res Rev. 2019. 49: 125-143.

12. CG Liu, T Hao, TT Yang, et al. The preliminary study on the diagnostic value of plasma exosomes microRNA-29c in the early diagnosis of the Alzheimer's disease. Chin J Lab Diagn. 2018; 22(5): 761-764.

13. Sha S, Shen X, Cao Y, et al. Mesenchymal stem cells-derived extracellular vesicles ameliorate Alzheimer's disease in rat models via the microRNA-29c3p/BACE1 axis and the Wnt/ $\beta$-catenin pathway. Aging (Albany NY). 2021; 13(11): 15285-15306.

14. Jia L, Qiu Q, Zhang H, Chu L, Du Y, Zhang J, Zhou C, Liang F, Shi S, Wang S, Qin W, Wang Q, Li F, Wang Q, Li Y, Shen L, Wei Y, Jia J. Concordance between the assessment of A 42 , T-tau, and P-T181-tau in peripheral blood neuronal-derived exosomes and cerebrospinal fluid. Alzheimers Dement. 2019; 15(8): 1071-1080.

15. McKhann GM, Knopman DS, Chertkow H, Hyman BT, Jack CR Jr, Kawas CH, Klunk WE, Koroshetz WJ, Manly JJ, Mayeux R, Mohs RC, Morris JC, Rossor MN, Scheltens P, Carrillo MC, Thies B, Weintraub S, Phelps CH. The diagnosis of dementia due to Alzheimer's disease: recommendations from the National Institute on Aging-Alzheimer's Association workgroups on diagnostic guidelines for Alzheimer's disease. Alzheimers Dement. 2011; 7(3): 263-269.

16. Gauthier S, Reisberg B, Zaudig M, Petersen RC, Ritchie K, Broich K, Belleville S, Brodaty H, Bennett D, Chertkow H, Cummings JL, de Leon M, Feldman H, Ganguli M, Hampel H, Scheltens P, Tierney MC, Whitehouse P, Winblad B; International Psychogeriatric Association Expert Conference on mild cognitive impairment. Mild cognitive impairment. Lancet. 2006; 367(9518): 1262-1270.

17. Molinuevo JL, Rabin LA, Amariglio R, Buckley R, Dubois B, Ellis KA, Ewers M, Hampel H, Klöppel S, Rami L, Reisberg B, Saykin AJ, Sikkes S, Smart CM, Snitz BE, Sperling R, van der Flier WM, Wagner M, Jessen F; Subjective Cognitive Decline Initiative (SCD-I) Working Group. Implementation of subjective cognitive decline criteria in research studies. Alzheimers Dement. 2017; 13(3): 296-311.

18. Koppara A, Wagner M, Lange C, Ernst A, Wiese B, König HH, Brettschneider C, Riedel-Heller S, Luppa M, Weyerer S, Werle J, Bickel H, Mösch E, Pentzek M, Fuchs A, Wolfsgruber S, Beauducel A, Scherer M, Maier W, Jessen F. Cognitive performance before and after the onset of subjective cognitive decline in old age. Alzheimers Dement (Amst). 2015; 1(2): 194-205.

19. Román GC, Tatemichi TK, Erkinjuntti T, Cummings JL, Masdeu JC, Garcia JH, Amaducci L, Orgogozo JM, Brun A, Hofman A, et al. Vascular dementia: diagnostic criteria for research studies. Report of the NINDS-AIREN International Workshop. Neurology. 1993; 43(2):250-260.

20. Kalaria RN. Neuropathological diagnosis of vascular cognitive impairment and vascular dementia with implications for Alzheimer's disease. Acta Neuropathol. 2016; 131(5): 659-685.

21. Teunissen CE, Petzold A, Bennett JL, Berven FS, Brundin L, Comabella M, Franciotta D, Frederiksen JL, Fleming JO, Furlan R, Hintzen RQ, Hughes SG, Johnson MH, Krasulova E, Kuhle J, Magnone MC, Rajda C, Rejdak K, Schmidt HK, van Pesch V, Waubant E, Wolf C, Giovannoni G, Hemmer B, Tumani H, Deisenhammer F. A consensus protocol for the standardization of cerebrospinal fluid collection and biobanking. Neurology. 2009; 73(22): 1914-1922.

22. Fiandaca MS, Kapogiannis D, Mapstone M, Boxer A, Eitan E, Schwartz JB, Abner EL, Petersen RC, Federoff HJ, Miller BL, Goetzl EJ. Identification of preclinical Alzheimer's disease by a profile of pathogenic proteins in neurally derived blood exosomes: A case-control study. Alzheimers Dement. 2015; 11(6): 600-607. 
23. Muller L, Hong CS, Stolz DB, Watkins SC, Whiteside TL. Isolation of biologically-active exosomes from human plasma. J Immunol Methods. 2014; 411 : 55-65.

24. Livak KJ, Schmittgen TD. Analysis of relative gene expression data using real-time quantitative PCR and the 2(-Delta Delta C(T)) Method. Methods. 2001; 25(4): 402-408.

25. Zhang J, Li S, Li L, Li M, Guo C, Yao J, Mi S. Exosome and exosomal microRNA: trafficking, sorting, and function. Genomics Proteomics Bioinformatics. 2015; 13(1): 17-24.

26. Liu CG, Zhao Y, Lu Y, Wang PC. ABCA1-labeled exosomes in serum contain higher microRNA-193b levels in Alzheimer's disease. Biomed Res Int. 2021; 2021: 5450397.

27. Qian H, Zhao X, Cao P, Lei J, Yan N, Gong X. Structure of the human lipid exporter amphiphysin 1. Cell. 2017; 169(7): 1228-1239. e10.

28. Rawat V, Wang S, Sima J, Bar R, Liraz O, Gundimeda U, Parekh T, Chan J, Johansson JO, Tang C, Chui HC, Harrington MG, Michaelson DM, Yassine HN. ApoE4 alters amphiphysin 1 membrane trafficking in astrocytes. J Neurosci. 2019; 39(48): 9611-9622.

29. Koldamova R, Fitz NF, Lefterov I. ATP-binding cassette transporter A1: from metabolism to neurodegeneration. Neurobiol Dis. $2014 ; 72$ Pt A: $13-21$.

\section{Figures}

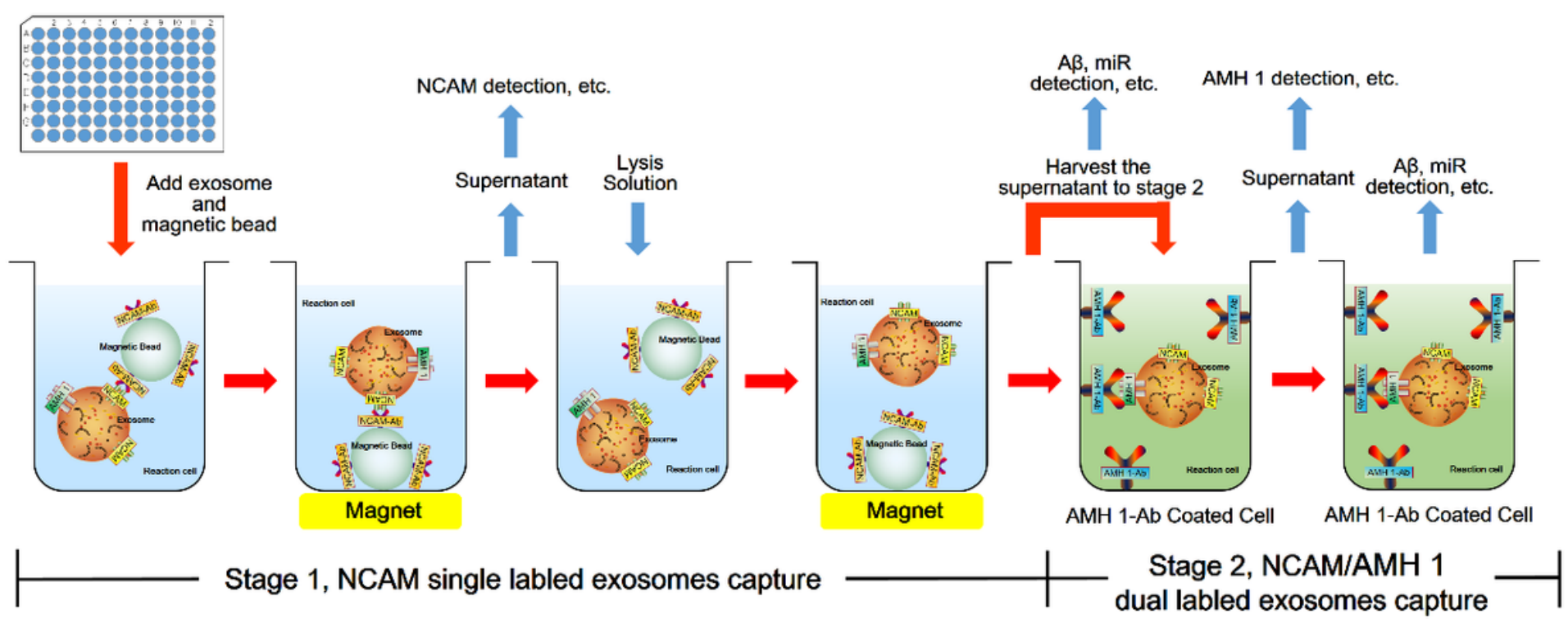

Figure 1

Procedure for collecting neuronal-derived exosomes from plasma. Stage 1 was used for NCAM single-labeled exosomes capture. Stage 1 and 2 were used together for NCAM/amphiphysin 1 dual-labeled exosomes capture. AMH 1 = amphiphysin 1. 
A

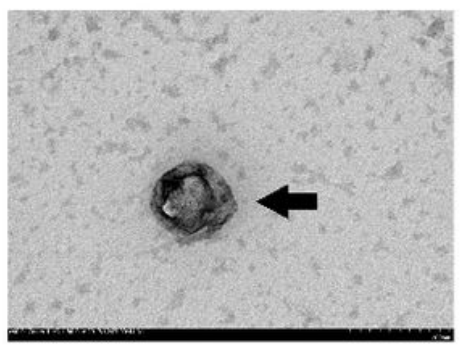

$\mathbf{F}$

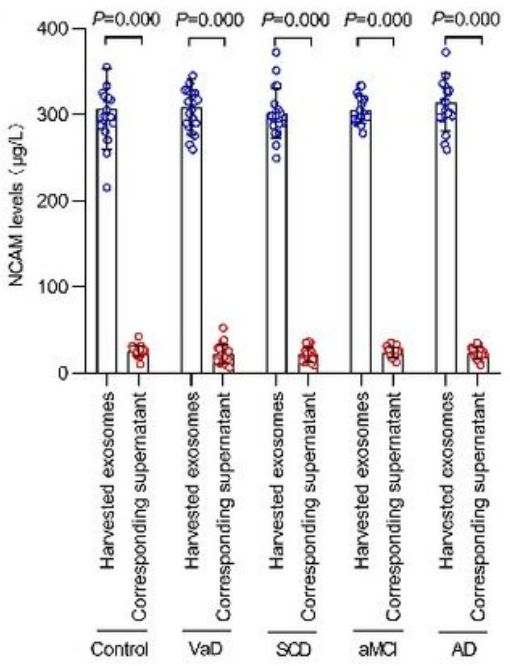

B

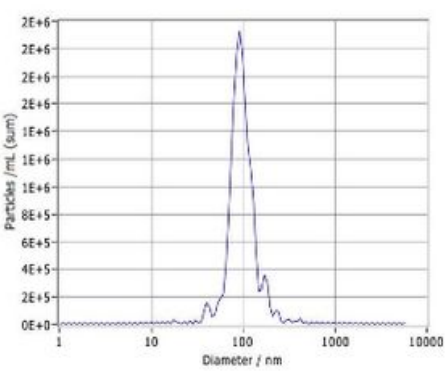

E

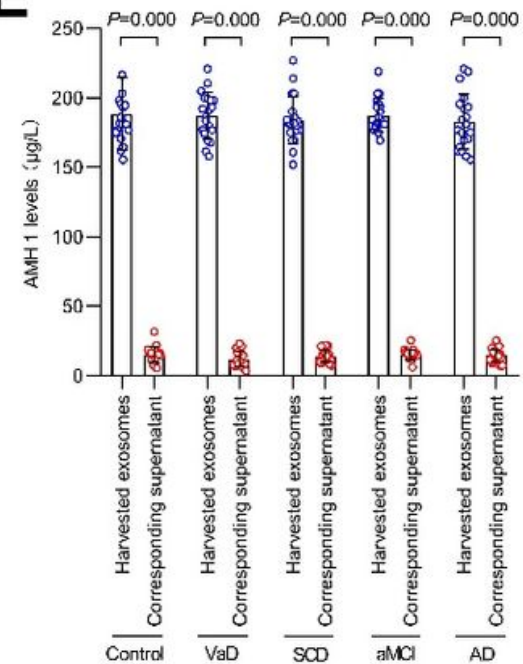

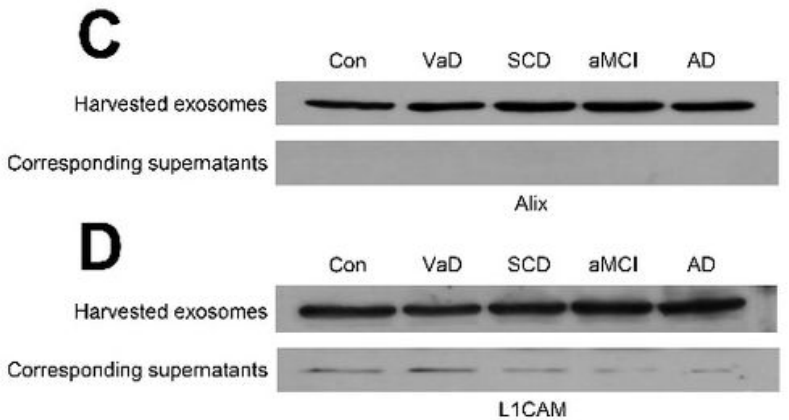

G

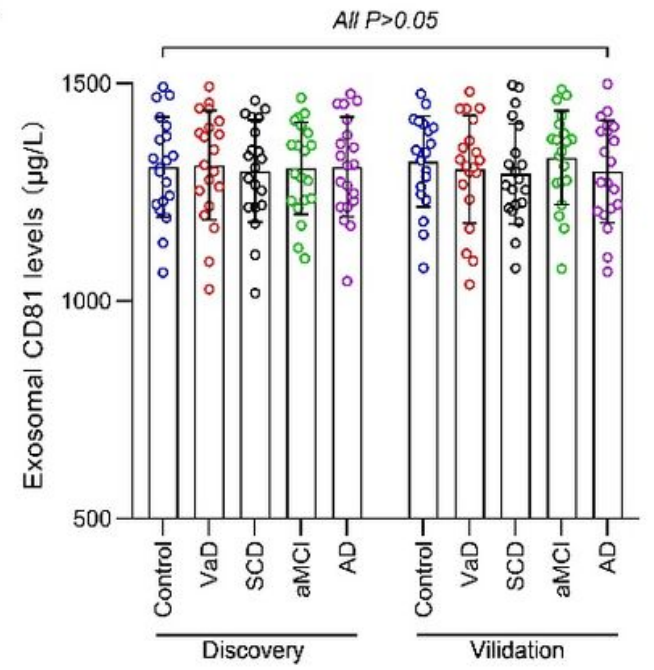

Figure 2

Confirmation of exosomal collection by TEM and Western blot and normalization of exosomal content. A: Representative TEM image showing neuronalderived exosomes (black arrow) that were successfully collected. B: Laser scattering microscopy result show that the size of the extract was consistent with the general size of exosomes. C-D: Representative western blot images showing that the exosomal marker Alix (C) and L1CAM (D) were highly expressed in exosomal samples but not detected or very low in corresponding supernatants. E-F: ELISA in controls, VaD, SCD, aMCl, and AD patients in the discovery stage show that the NCAM (E) and amphiphysin 1 (F) concentration in captured exosomes were increased by approximately 10-fold compared with that in corresponding supernatants. G: CD81 was measured to normalize the exosomal content. No difference was detected between each group. $\mathrm{n}=20$ in each group. The $\mathrm{P}$ values are shown in the figure. $\mathrm{AMH} 1$ = amphiphysin 1. 

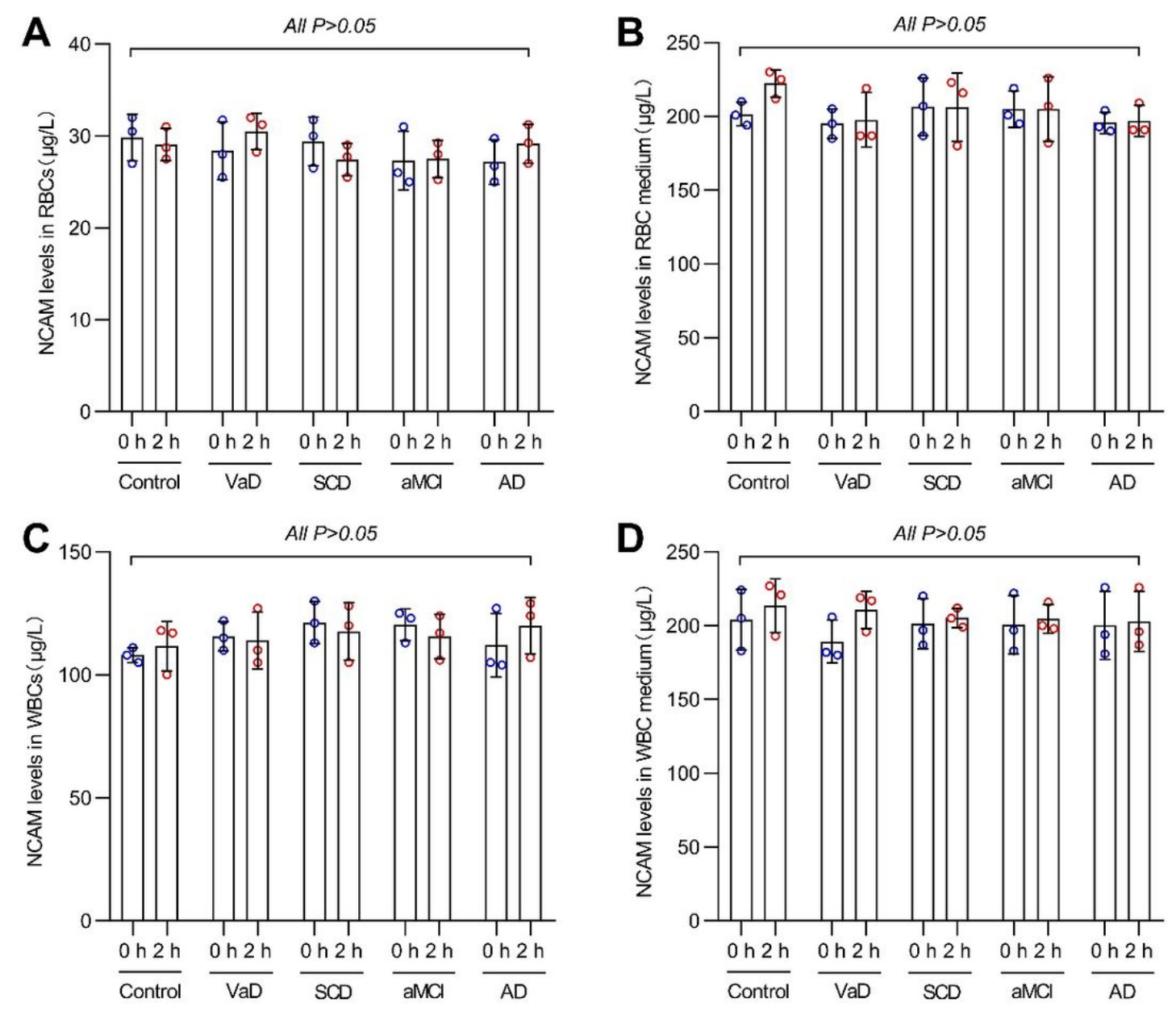

Figure 3

WBC and RBC NCAM secretion experiment results. A-B: After 2 hours of incubation, NCAM levels in WBCs and RBCs did not decrease significantly (all P>0.05). C-D: After 2 hours of cultivation, the NCAM levels in WBC and RBC medium did not increase significantly (all $P>0.05)$. $n=3$ in each group. 
A

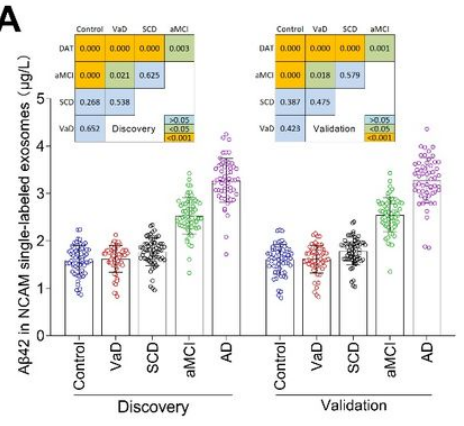

C

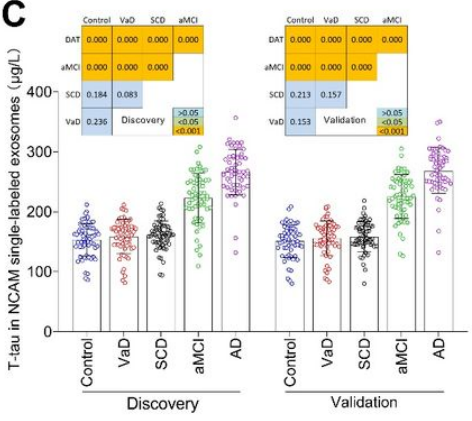

\section{E}

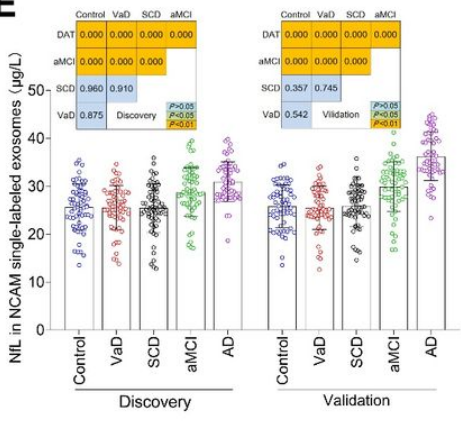

B

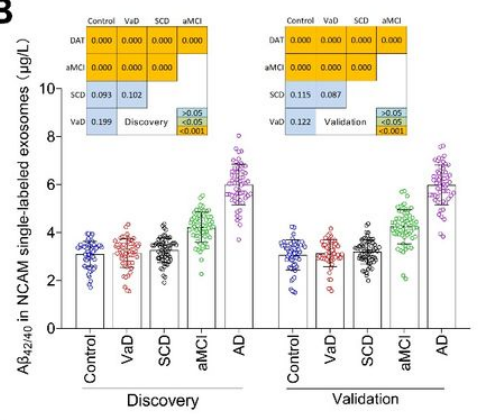

D

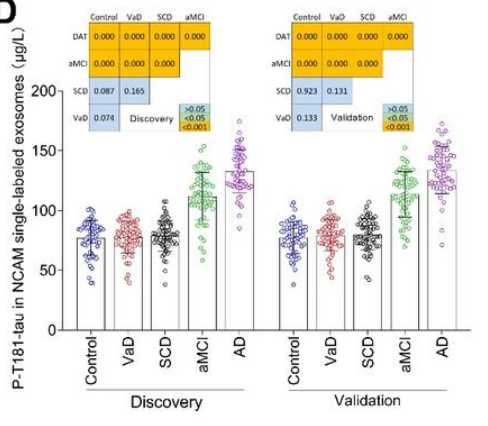

F

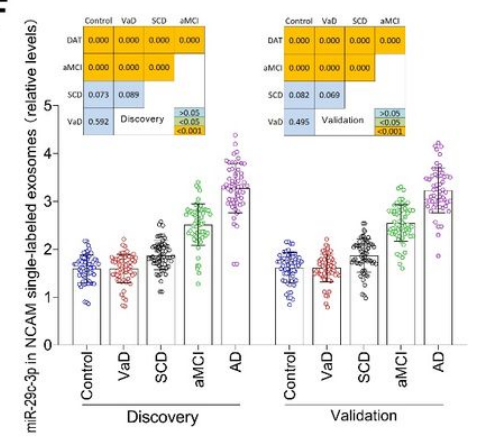

\section{Figure 4}

Levels of $A \beta 42(A), A \beta 42 / 40(B)$, T-tau (C), P-T181-tau (D), NfL (E), and miR-29c-3p (F) in NCAM single-labeled exosomes of plasma. In the discovery stage, $n=$ 30 (Control), 30 (VaD), 45 (SCD), 50 (aMCl), $40(\mathrm{AD})$. In the validation stage, $n=30$ (Control), $30(\mathrm{VaD}), 47(\mathrm{SCD}), 45(\mathrm{aMCl}), 45(\mathrm{AD})$. The P values are shown in the figure. 

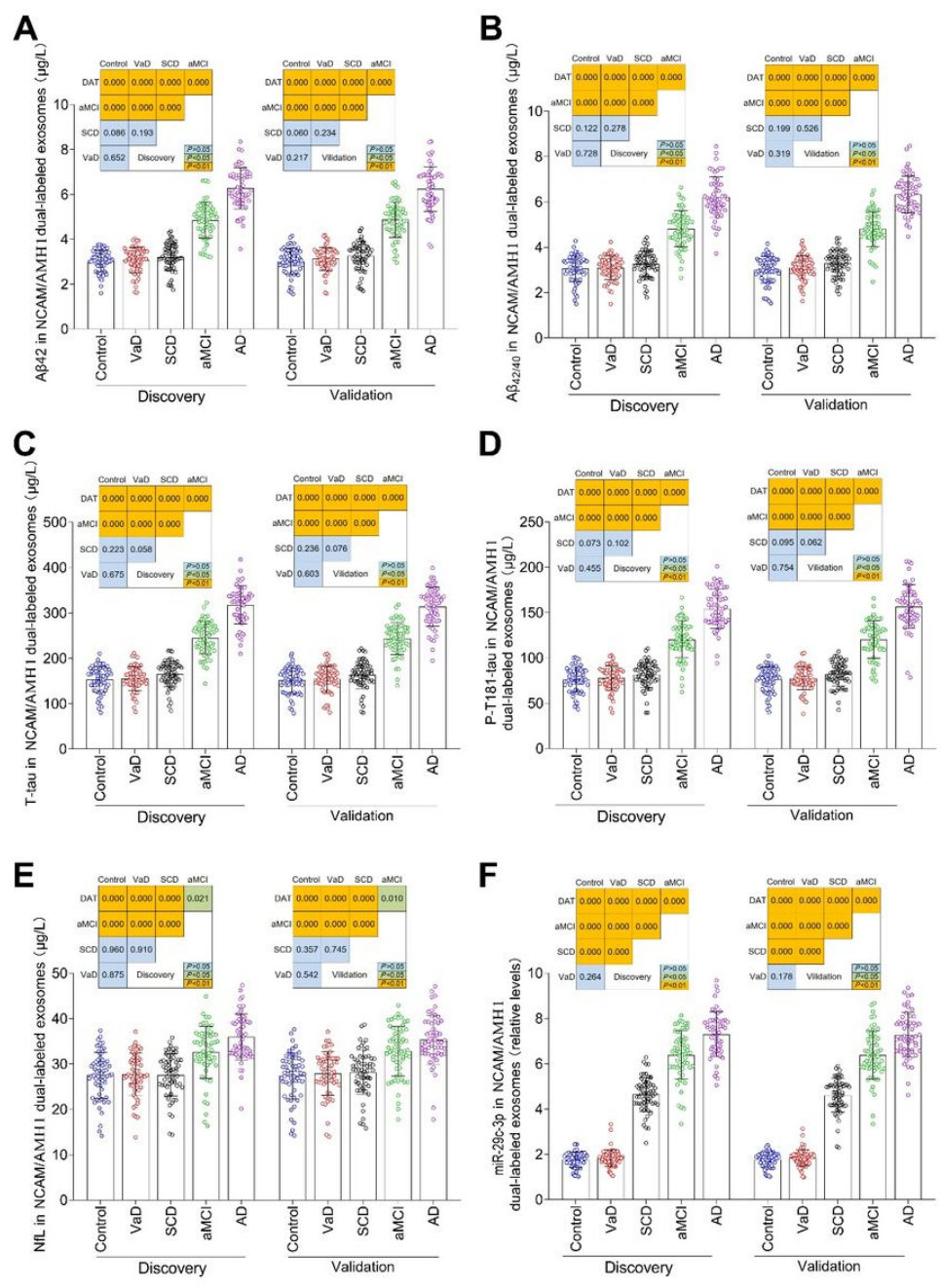

\section{Figure 5}

Levels of $A \beta 42(A), A \beta 42 / 40$ (B), T-tau (C), p-T181-tau (D), NfL (E), and miR-29c-3p (F) in NCAM/amphiphysin 1 dual-labeled exosomes of plasma. In the discovery stage, $n=30$ (Control), $30(\mathrm{VaD}), 45(\mathrm{SCD}), 50$ (aMCl), $40(\mathrm{AD})$. In the validation stage, $n=30$ (Control), $30(\mathrm{VaD}), 47(\mathrm{SCD}), 45(\mathrm{aMCl}), 45(\mathrm{AD})$. The $\mathrm{P}$ values are shown in the figure. AMH $1=$ amphiphysin 1.

\begin{tabular}{|c|c|c|c|c|c|c|c|c|c|}
\hline & \multicolumn{4}{|c|}{$\mathrm{NE}$} & \multicolumn{4}{|c|}{ NAE } & \\
\hline & $\mathrm{VaD}$ & $S C D$ & $\mathrm{aMCl}$ & $A D$ & $\mathrm{VaD}$ & $S C D$ & $\mathrm{aMCl}$ & $A D$ & \\
\hline$A B 42$ & 1.00 & 1.09 & 1.51 & 1.80 & 1.03 & 1.57 & 1.71 & 2.07 & 4 \\
\hline $\mathrm{A} \beta_{42 / 40}$ & 1.02 & 1.03 & 1.31 & 1.42 & 1.02 & 1.08 & 1.59 & 2.07 & \\
\hline T-tau & 1.03 & 1.05 & 1.47 & 1.43 & 1.01 & 1.08 & 1.50 & 1.87 & 3 \\
\hline T181-tau & 1.02 & 1.03 & 1.46 & 1.72 & 1.02 & 1.07 & 1.58 & 1.95 & \\
\hline NfL & 0.99 & 1.00 & 1.14 & 1.31 & 1.01 & 1.02 & 1.19 & 1.30 & 2 \\
\hline miR-384 & 1.00 & 1.17 & 1.58 & 2.03 & 1.05 & 2.63 & 3.63 & 4.14 & \\
\hline
\end{tabular}

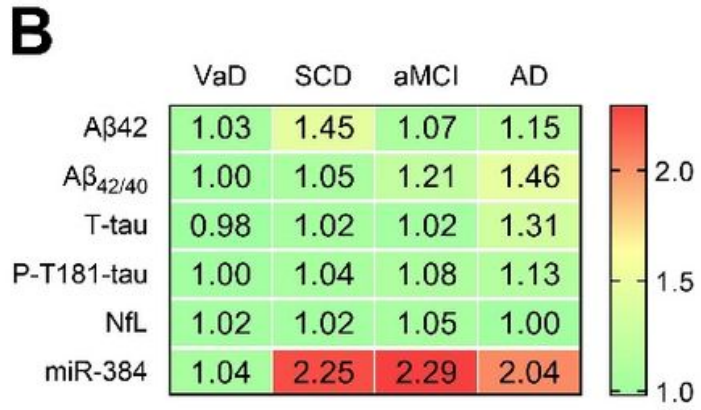

The ratio of NAE to NE

\section{Figure 6}

The fold changes of biomarkers. A shows the ratio of each biomarker relative to the control group in NCAM single-labeled exosomes (NE) and NCAM/amphiphysin 1 dual-labeled exosomes (NAM) from VaD, SCD, aMCl, and AD. B shows the ratio of NAE to NAM. 

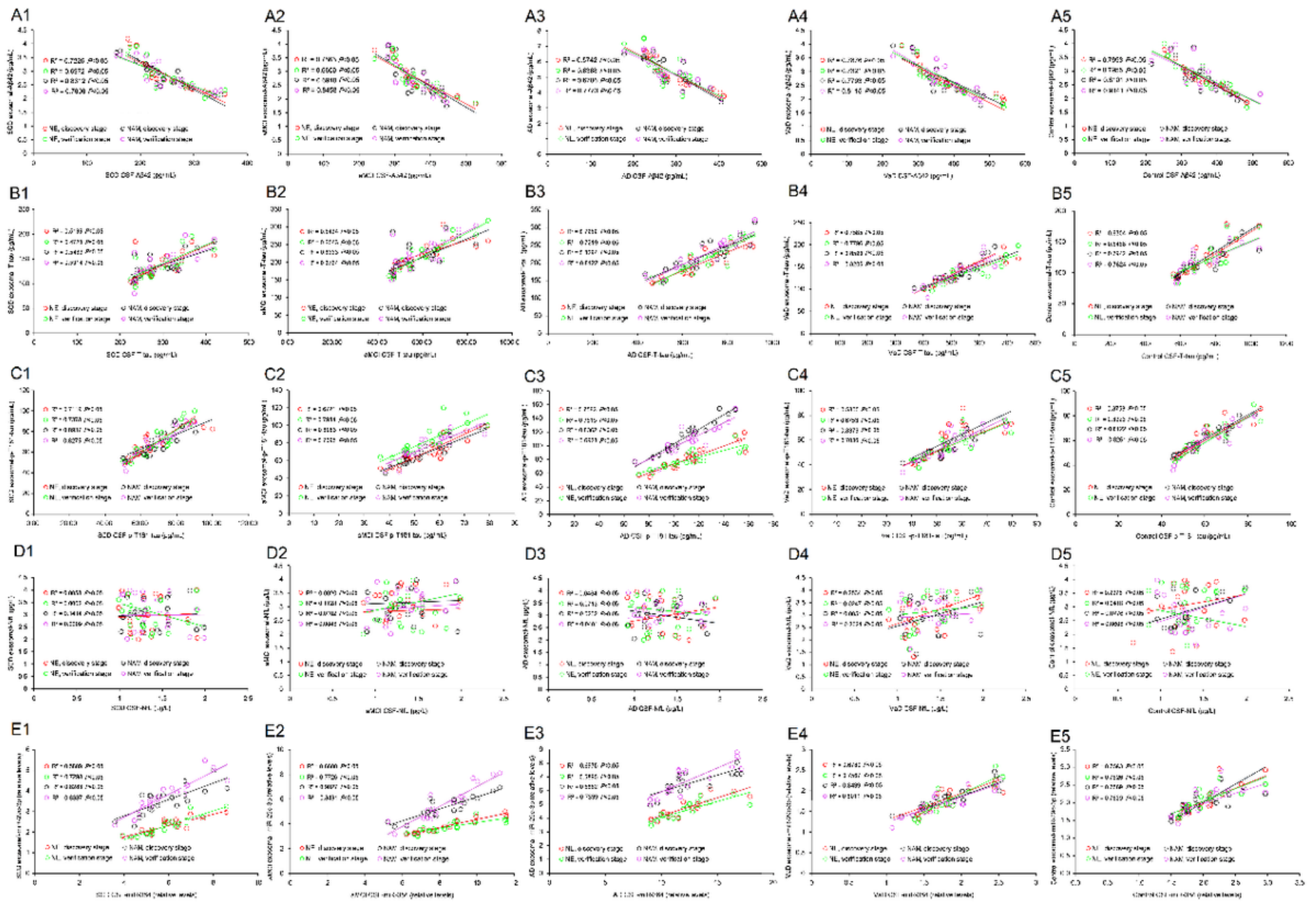

\section{Figure 7}

The levels of exosomal biomarkers are significantly correlated with the levels of CSF biomarkers. A, C and E show robust correlations of AB42 (A), P-T181-tau (C), and miR-29c-3p (E) from exosomes and CSF in SCD (1), aMCI (2), AD (3), VaD (4), and controls (5) (all P<0.05). B show significant correlations of T-tau from exosomes and CSF in SCD (1), aMCl (2), AD (3), VaD (4), and controls (5) (all P<0.05). C show no correlation of NfL from exosomes and CSF in SCD (1), $\mathrm{aMCl}(2), \mathrm{AD}(3), \operatorname{VaD}(4)$, and controls (5) (all P>0.05). $\mathrm{n}=20$ in each group. NE = NCAM single-labeled exosomes, NAM = NCAM/amphiphysin 1 dual-labeled exosomes. 
A
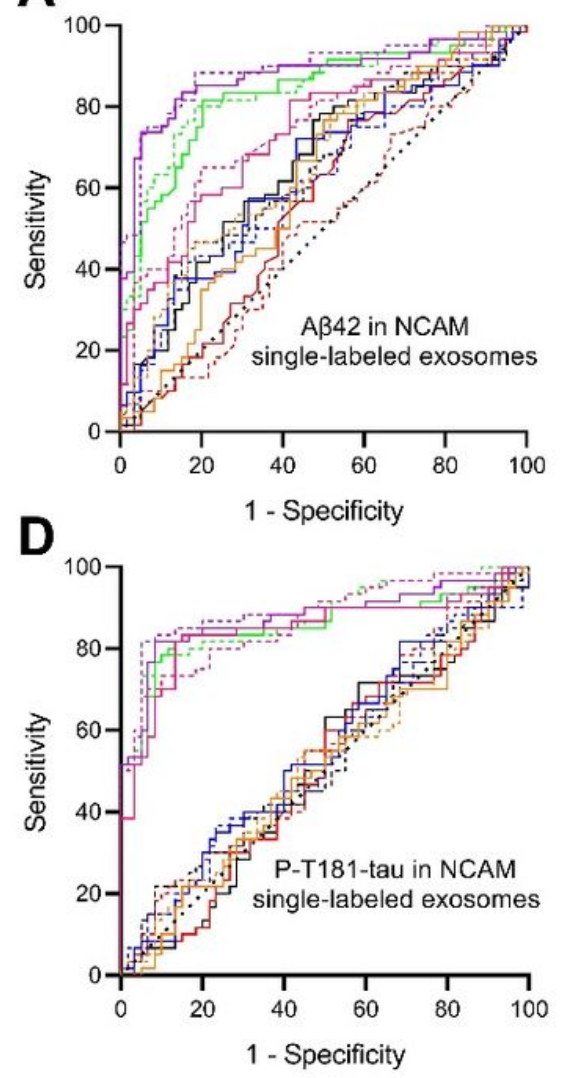

B

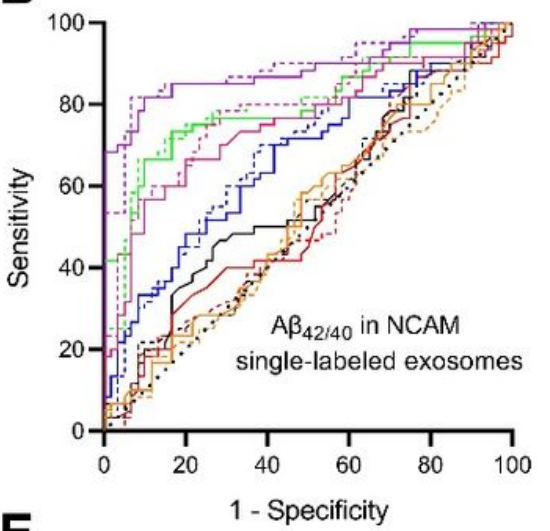

E

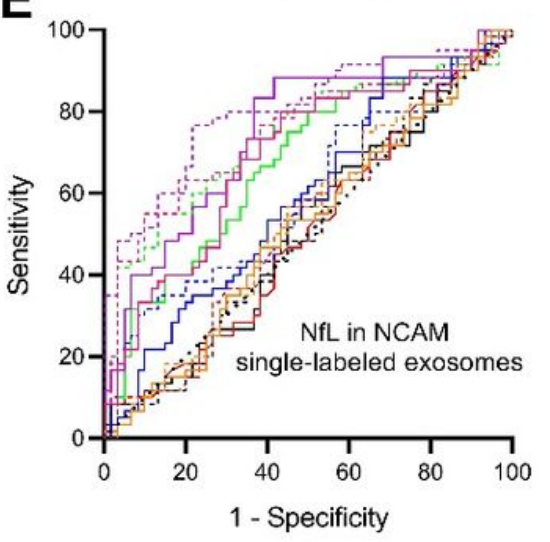

C

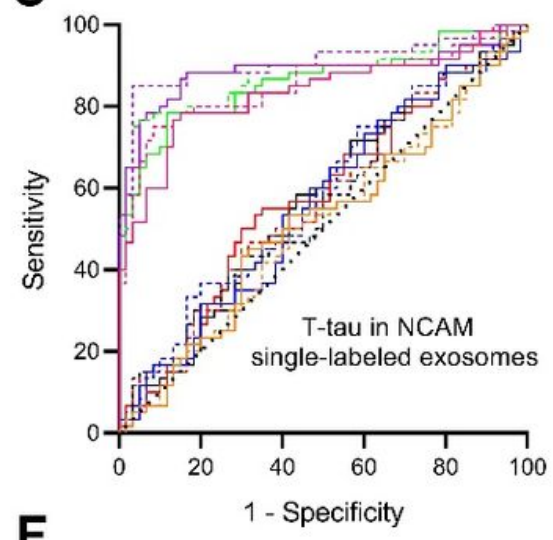

$\mathbf{F}$

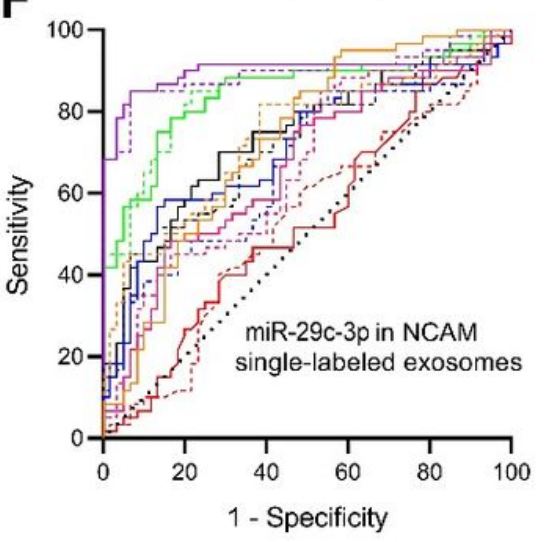

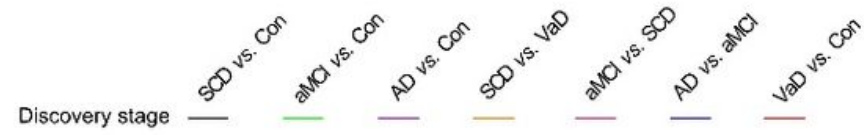

Verification stage

\section{Figure 8}

Diagnostic power analysis of plasma exosomal biomarkers in NCAM single-labeled exosomes of plasma. A-F show multiple ROC analyses of AB42 (A), A $42 / 40$ (B), T-tau (C), P-T181-tau (D), NfL (E), and miR-29c-3p (F) in NCAM single-labeled exosomes of plasma in SCD, aMCl, AD, VaD, and controls. In the discovery stage, $n=30$ (Control), $30(\mathrm{VaD}), 45(\mathrm{SCD}), 50$ (aMCl), $40(\mathrm{AD})$. In the validation stage, $n=30$ (Control), $30(\mathrm{VaD}), 47(\mathrm{SCD}), 45(\mathrm{aMCl}), 45(\mathrm{AD})$. The $P$ values are shown in the figure. Con $=$ control. 

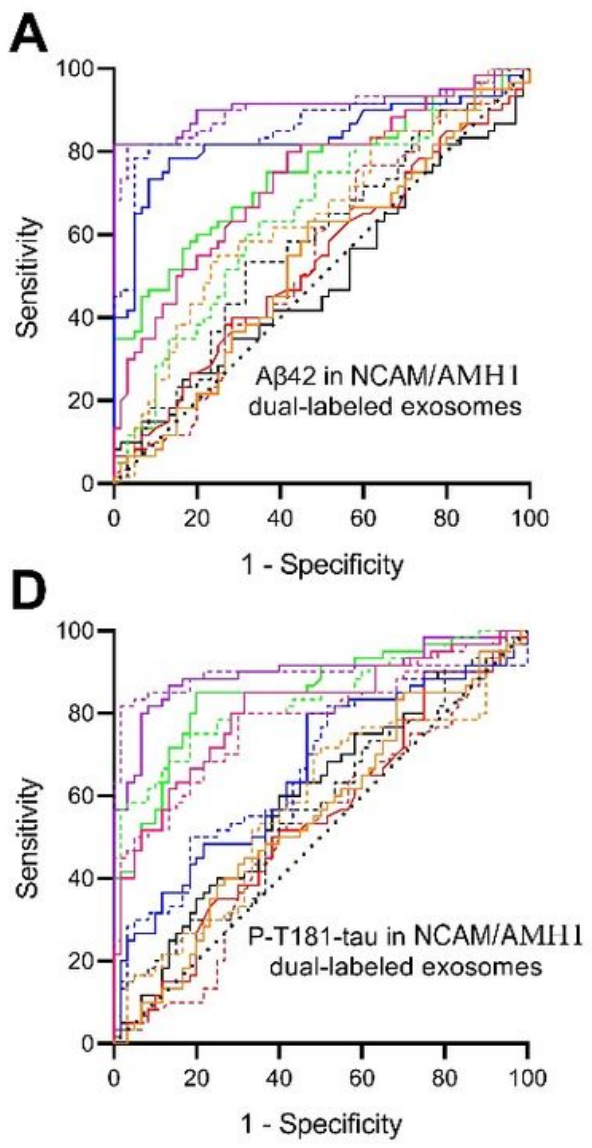

B

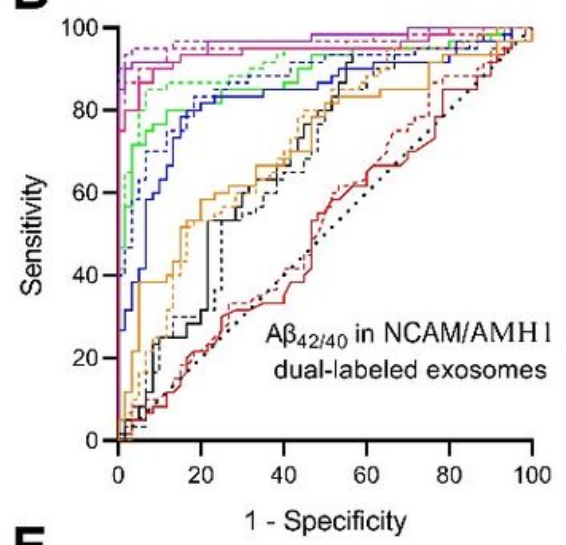

E

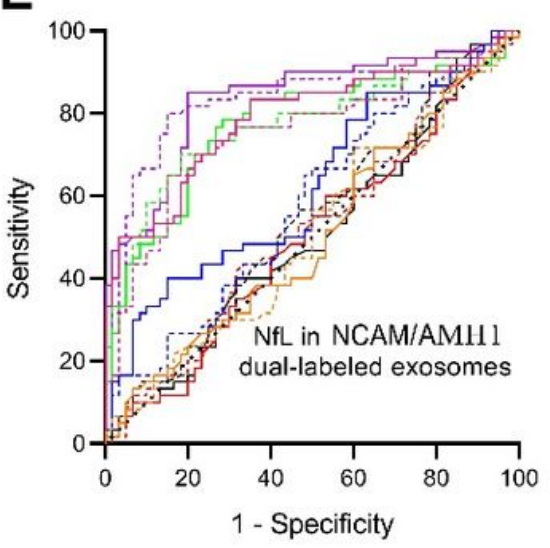

C
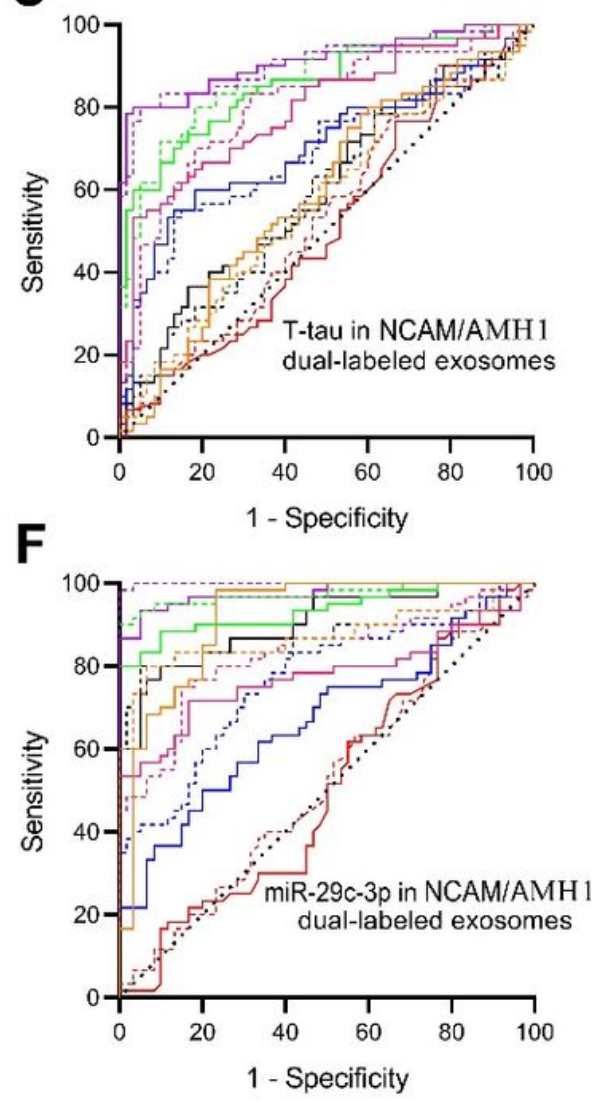

Dbsoverystase

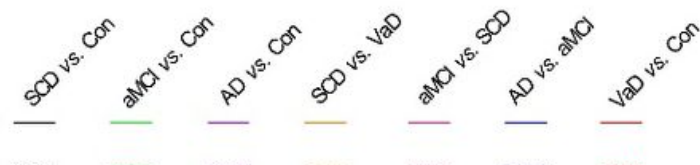

Verification stage .....

\section{Figure 9}

Diagnostic power analysis of plasma exosomal biomarkers in NCAM/amphiphysin 1 dual-labeled exosomes of plasma. A-F show multiple ROC analyses of Aß42 (A), Aß42/40 (B), T-tau (C), P-T181-tau (D), NfL (E), and miR-29c-3p (F) in NCAM/amphiphysin 1 dual-labeled exosomes of plasma in SCD, aMCl, AD, $\mathrm{VaD}$, and controls. In the discovery stage, $\mathrm{n}=30$ (Control), 30 (VaD), 45 (SCD), 50 (aMCl), $40(\mathrm{AD})$. In the validation stage, $n=30$ (Control), 30 (VaD), $47(\mathrm{SCD})$, $45(\mathrm{aMCl}), 45(\mathrm{AD})$. The $\mathrm{P}$ values are shown in the figure. Con = control, $\mathrm{AMH} 1=\operatorname{amphiphysin} 1$.

A

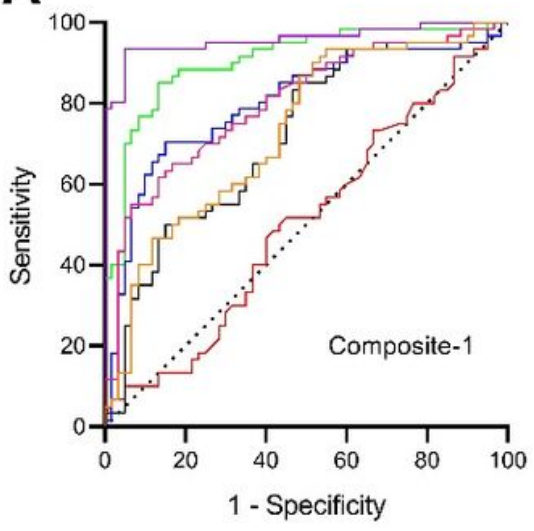

B

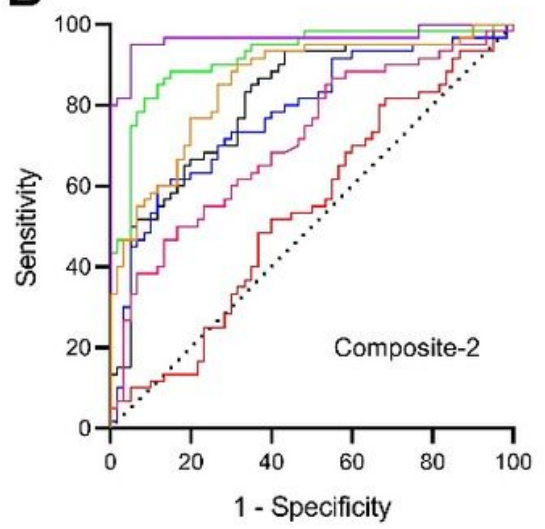

C

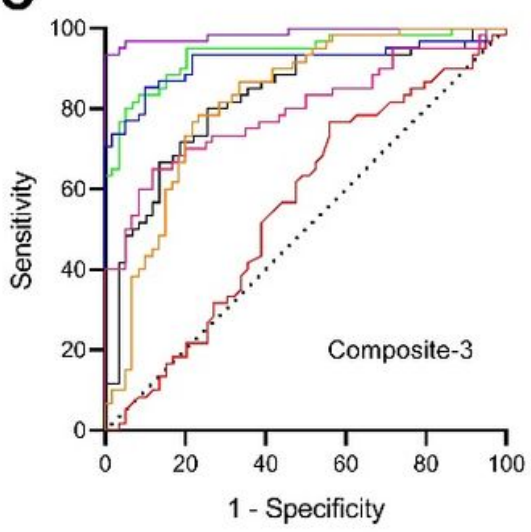

Figure 10

Higher performance of the combined biomarkers. The data of the discovery and validation stages was integrated for analysis. The performances of combined analysis of AB42, AB42/40, T-tau, P-T181-tau, NfL, and miR-29c-3p in plasma NCAM single-labeled exosomes, plasma NCAM/amphiphysin 1 dual-labeled exosomes or CSF as composite-1 (A), composite-2 (B), and composite-3 (C) respectively. $n=60$ (Control), 60 (VaD), 92 (SCD), 95 (aMCl), 85 (AD). 